\title{
ML 3.0 Smoothed Aggregation User's
}

Guide

Marzio Sala, Jonathan J. Hu, Ray S. Tuminaro

\author{
Prepared by \\ Sandia National Laboratories \\ Albuquerque, New Mexico 87185 and Livermore, California $\$ 4550$ \\ Sandia is a multiprogram laboratory operated by Sandia Corporation, \\ a Lockheed Martin Company, for the United States Department of Energy's \\ National Nuclear Security Administration under Contract DE-AC04-94AL85000.
}

Approved for public release; further dissemination unlimited.

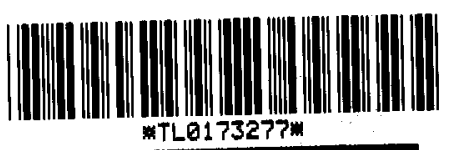

SANDIA NATIONAL

LABORATORIES

TECHNICAL LIBRARY

\section{Sandia National Laboratories}

\author{
LBRARY DOCUMENT \\ DU WOT DESTROY \\ RETUWH TO \\ LIBRARY VAULT
}


Issued by Sandia National Laboratories, operated for the United States Department of Energy by Sandia Corporation.

NOTICE: This report was prepared as an account of work sponsored by an agency of the United States Government. Neither the United States Government, nor any agency thereof, nor any of their employees, nor any of their contractors, subcontractors, or their employees, make any warranty, express or implied, or assume any legal liability or responsibility for the accuracy, completeness, or usefulness of any information, apparatus, product, or process disclosed, or represent that its use would not infringe privately owned rights. Reference herein to any specific commercial product, process, or service by trade name, trademark, manufacturer, or otherwise, does not necessarily constitute or imply its endorsement, recommendation, or favoring by the United States Government, any agency thereof, or any of their contractors or subcontractors. The views and opinions expressed herein do not necessarily state or reflect those of the United States Government, any agency thereof, or any of their contractors.

Printed in the United States of America. This report has been reproduced directly from the best available copy.

Available to DOE and DOE contractors from

U.S. Department of Energy

Office of Scientific and Technical Information

P.O. Box 62

Dak Ridge, TN 37831

Telephone: (865)576-8401

Facsimile: (865)576-5728

E-Mail: reports@adonis.osti.gov

Online ordering: http://www.doe.gov/bridge

Available to the public from

U.S. Department of Commerce

National Technical Information Service

5285 Port Royal Rd

Springfield, VA 22161

Telephone: (800)553-6847

Facsimile: (703)605-6900

E-Mail: $\quad$ orders@ntis.fedworld.gov

Online order: http://www.ntis.gov/help/ordermethods.asp?loc=7-4-0\# online

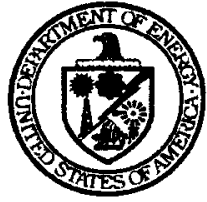


SAND2004-2195

Unlimited Release

Printed May 2004

\title{
ML 3.0 Smoothed Aggregation User's Guide
}

\author{
Marzio Sala \\ Computational Math \& Algorithms \\ Sandia National Laboratories \\ P.O. Box 5800 \\ Albuquerque, NM 87185-1110 \\ Jonathan J. Hu and Ray S. Tuminaro \\ Computational Math \& Algorithms \\ Sandia National Laboratories \\ P.O. Box 0969 \\ Livermore, CA 94551-0969

\section{LIBRARY DOCUMENT DO NOT DESTROY RETURN TO LIBRARY VAULT}

\begin{abstract}
ML is a multigrid preconditioning package intended to solve linear systems of equations $A x=b$ where $A$ is a user supplied $n \times n$ sparse matrix, $b$ is a user supplied vector of length $n$ and $x$ is a vector of length $n$ to be computed. ML should be used on large sparse linear systems arising from partial differential equation (PDE) discretizations. While technically any linear system can be considered, ML should be used on linear systems that correspond to things that work well with multigrid methods (e.g. elliptic PDEs). ML can be used as a stand-alone package or to generate preconditioners for a traditional iterative solver package (e.g. Krylov methods). We have supplied support for working with the AzTEC 2.1 and AzTECOO iterative package [15]. However, other solvers can be used by supplying a few functions.

This document describes one specific algebraic multigrid approach: smoothed aggregation. This approach is used within several specialized multigrid methods: one for the eddy current formulation for Maxwell's equations, and a multilevel and domain decomposition method for symmetric and nonsymmetric systems of equations (like elliptic equations, or compressible and incompressible fluid dynamics problems). Other methods exist within ML but are not described in this document. Examples are given illustrating the problem definition and exercising multigrid options.
\end{abstract}


(page intentionally left blank) 


\section{Contents}

$\begin{array}{lll}1 & \text { Notational Conventions } & 7\end{array}$

$\begin{array}{llr}2 & \text { Overview } & 7\end{array}$

$\begin{array}{lll}3 & \text { Multigrid Background } & 7\end{array}$

4 Configuring and Building ML $\quad 8$

4.1 Building in Standalone Mode . . . . . . . . . . . . . . . . . . 9

4.2 Building with AzTEC 2.1 Support . . . . . . . . . . . . . 9

4.3 Building with TRILINOS Support (RECOMMENDED) . . . . . . . . . . 9

4.3.1 Enabling Third Party Library Support . . . . . . . . . . . . . 10

4.3 .2 Enabling Profiling. . . . . . . . . . . . . 11

5 ML and Epetra: Getting Started with the MultiLevelPreconditioner Class 12

5.1 Example 1: ml_example_epetra_preconditioner.cpp . . . . . . . . . 12

5.2 Example 2: ml_example_epetra_preconditioner_2level.cpp . . . . . . . . . . 14

6 Parameters for the ML_Epetra::MultiLevelPreconditioner Class 15

6.1 Setting Options on a Specific Level . . . . . . . . . . . . . . . . 16

6.2 General Usage of the Parameter List . . . . . . . . . . . . . . . . . . 16

6.3 Default Parameter Settings for Common Problem Types . . . . . . . . . . . 17

6.4 Commonly Used Parameters . . . . . . . . . . . . . . . . . . . . . . . 19

6.5 List of All Parameters for MultiLevelPreconditioner Class . . . . . . . . . . . 19

6.5 .1 General Options . . . . . . . . . . . . . . . . . 19

6.5 .2 Aggregation Parameters . . . . . . . . . . . . 21

6.5.3 Smoothing Parameters . . . . . . . . . . . . . . . . 21

6.5.4 Coarsest Grid Parameters . . . . . . . . . . . . . . . 22

7 Advanced Usage of ML $\quad 23$

8 Multigrid \& Smoothing Options $\quad 24$

9 Smoothed Aggregation Options $\quad 26$

9.1 Aggregation Options . . . . . . . . . . . . . . . . 26

9.2 Interpolation Options . . . . . . . . . . . . . 27

10 Advanced Usage of ML and Epetra 28

11 Using ML without Epetra $\quad 29$

11.1 Creating a ML matrix: Single Processor . . . . . . . . . . . . . . . . . . . 29

11.2 Creating a ML matrix: Multiple Processors . . . . . . . . . . . . 31

12 Visualization Capabilities $\quad 34$ 
13 ML Functions $\quad 36$

AZ_ML_Set_Amat . . . . . . . . . . . . . . . . . . 36

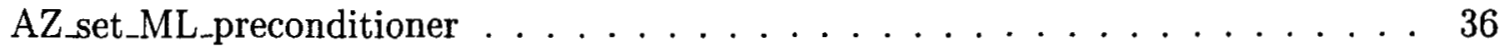

ML_Aggregate_Create . . . . . . . . . . . . . . . . 37

ML_Aggregate_Destroy . . . . . . . . . . . . . . . . . . . . . 37

ML_Aggregate_Set_CoarsenScheme_Coupled . . . . . . . . . . . . . . . . 38

ML_Aggregate_Set_CoarsenScheme_MIS . . . . . . . . . . . . . . . . . 38

ML_Aggregate_Set_CoarsenScheme_Uncoupled . . . . . . . . . . . . . . . . 39

ML_Aggregate_Set_CoarsenScheme_METIS . . . . . . . . . . . . . . . . . . 39

ML_Aggregate_Set_CoarsenScheme_ParMETIS . . . . . . . . . . . . . . . . 40

ML_Aggregate_Set_DampingFactor . . . . . . . . . . . . . . . . . . . . 40

ML_Aggregate_Set_MaxCoarseSize . . . . . . . . . . . . . . . . . . . 41

ML_Aggregate_Set_NullSpace . . . . . . . . . . . . . . . . . . 41

ML_Aggregate_Set_SpectralNormScheme_Calc . . . . . . . . . . . . . . . . 42

ML_Aggregate_Set_SpectralNormScheme_Anorm . . . . . . . . . . . . . 42

ML_Aggregate_Set_Threshold . . . . . . . . . . . . . . 43

ML_Create . . . . . . . . . . . . . . . . . . . . . . . . . . . . . . . . . . . . .

ML_Destroy . . . . . . . . . . . . . . . . . . . . . 44

ML_Gen_Blocks_Aggregates . . . . . . . . . . . . . . . . . 44

ML_Gen_Blocks_Metis . . . . . . . . . . . . . . . . . . . . 45

ML_Gen_CoarseSolverSuperLU . . . . . . . . . . . . . . 45

ML_Gen_MGHierarchy_UsingAggregation . . . . . . . . . . . . . . . 46

ML_Gen_SmootherAmesos . . . . . . . . . . . . . . . . . . 47

ML_Gen_SmootherAztec . . . . . . . . . . . . . . . . . . . . . . . . . . . . . . .

ML_Gen_Smoother_BlockGaussSeidel . . . . . . . . . . . . . . . . . 48

ML_Gen_Smoother_GaussSeidel . . . . . . . . . . . . . . . . . 49

ML_Gen_Smoother_Jacobi . . . . . . . . . . . . . . . . . . 50

ML_Gen_Smoother_SymGaussSeidel . . . . . . . . . . . . . . . . 51

ML_Gen_Smoother_VBlockJacobi . . . . . . . . . . . . . . . . 51

ML_Gen_Smoother_VBlockSymGaussSeidel . . . . . . . . . . . . . . . . 52

ML_Gen_Solver . . . . . . . . . . . . . . . . . . . . 53

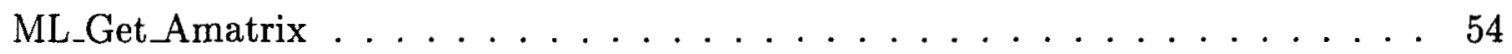

MLInit_Amatrix . . . . . . . . . . . . . . . . 54

MLIterate . . . . . . . . . . . . . . . . . . 55

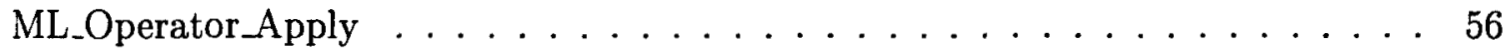

ML_Operator_Get_Diag . . . . . . . . . . . . . . . . . 56

ML_Operator_Getrow . . . . . . . . . . . . . . . . 57

ML_Set_Amatrix_Getrow . . . . . . . . . . . . . . . . . 58

ML_Set_Amatrix_Matvec . . . . . . . . . . . . . . . . . 58

ML_Set_ResidualOutputFrequency . . . . . . . . . . . . . . . . 59

ML_Set_Smoother . . . . . . . . . . . . . . . . . . . . 59

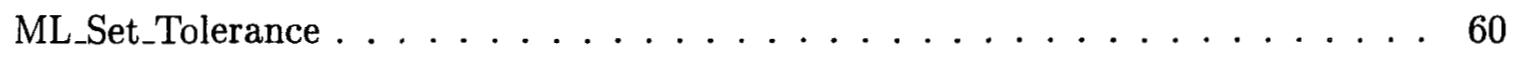

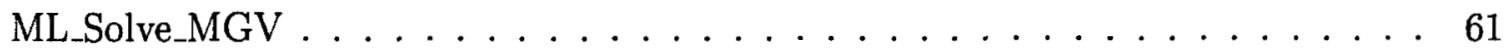




\section{Notational Conventions}

In this guide, we show typed commands in this font:

$\%$ a_really_long_command

The character $\%$ indicates any shell prompt ${ }^{1}$. Function names are shown as ML_Gen_Solver. Names of packages or libraries as reported in small caps, as EPETRA. Mathematical entities are shown in italics.

\section{Overview}

This guide describes the use of an algebraic multigrid method within the ML package. The algebraic multigrid method can be used to solve linear system systems of type

$$
A x=b
$$

where $A$ is a user supplied $n \times n$ sparse matrix, $b$ is a user supplied vector of length $n$ and $x$ is a vector of length $n$ to be computed. ML is intended to be used on (distributed) large sparse linear systems arising from partial differential equation (PDE) discretizations. While technically any linear system can be considered, ML should be used on linear systems that correspond to things that work well with multigrid methods (e.g. elliptic PDEs).

The ML package is used by creating a ML object and then associating a matrix, $A$, and a set of multigrid parameters which describe the specifics of the solver. Once created and initialized, the ML object can be used to solve linear systems.

This manual is structured as follows. Multigrid and multilevel methods are briefly recalled in Section 3. The process of configuring and building ML is outlined in Section 4. Section 5 shows the basic usage of ML as a black-box preconditioner for EPETRA matrices. The definition of (parallel) preconditioners using ML_Epetra::MultiLevelPreconditioner is detailed. This class only requires the linear system matrix, and a list of options. Available parameters for ML_Epetra::MultiLevelPreconditioner are reported in Section 6. More advanced uses of ML are presented in Section 7. Here, we present how to define and fine-tune smoothers, coarse grid solver, and the multilevel hierarchy. Multigrid options are reported in Section 8. Smoothing options are reported in Section 9, where we also present how to construct a user's defined smoother. Advanced usage of ML with EPETRA objects is reported in Section 10. Section 11 reports how to define matrices in ML format without depending on EPETRA. Section 12 detailes the (limited) visualization capabilities of ML.

\section{Multigrid Background}

A brief multigrid description is given (see [1], [6], or [7] for more information). A multigrid solver tries to approximate the original PDE problem of interest on a hierarchy of grids and use 'solutions' from coarse grids to accelerate the convergence on the finest grid. A simple multilevel iteration is illustrated in Figure 1. In the above method, the $S_{k}^{1}\left(\right.$ )'s and $S_{k}^{2}($ )'s

\footnotetext{
${ }^{1}$ For simplicity, commands are shown as they would be issued in a Linux or Unix environment. Note, however, that ML has and can be built successfully in a Windows environment.
} 


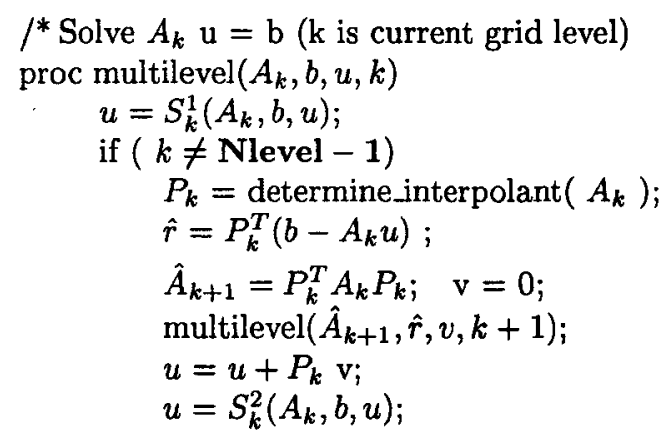

Figure 1: High level multigrid V cycle consisting of 'Nlevel' grids to solve (1), with $A_{0}=A$.

are approximate solvers corresponding to $k$ steps of pre and post smoothing, respectively. These smoothers are discussed in Section 8. For now, it suffices to view them as basic iterative methods (e.g. Gauss-Seidel) which effectively smooth out the error associated with the current approximate solution. The $P_{k}$ 's (interpolation operators that transfer solutions from coarse grids to finer grids) are the key ingredient that are determined automatically by the algebraic multigrid method ${ }^{2}$. For the purposes of this guide, it is important to understand that when the multigrid method is used, a hierarchy of grids, grid transfer operators $\left(P_{k}\right)$, and coarse grid discretizations $\left(A_{k}\right)$ are created. To complete the specification of the multigrid method, smoothers must be supplied on each level. There are several smoothers within ML or an iterative solver package can be used, or users can write their own smoother (see Section 8).

\section{Configuring and Building ML}

ML is configured and built using the GNU autoconf [4] and automake [5] tools. It can be configured and build as a standalone package without or with AZTEC 2.1 support (as detailed in Section 4.1 and 4.2), or as a part of the TRILINOS framework [8] (as described in Section 4.3). Even though ML can be compiled and used as a standalone package, the recommended approach is to build ML as part of the TRILINOs framework, as a richer set of features are then available.

ML has been configured and built successfully on a wide variety of operating systems, and with a variety of compilers (as reported in Table 1).

\begin{tabular}{|ll|}
\hline Operating System & Compilers(s) \\
\hline Linux & GNU and Intel \\
IRIX N32, IRIX 64, HPUX, Solaris, DEC & Native \\
ASCI Red & Native and Portland Group \\
CPlant & Native \\
Windows & Microsoft \\
\hline
\end{tabular}

Table 1: Main operating systems and relative compilers supported by ML.

\footnotetext{
${ }^{2}$ The $P_{k}$ 's are usually determined as a preprocessing step and not computed within the iteration.
} 
Although it is possible to configure directly in the ML home directory, we strongly advise against this. Instead, we suggest working in an independent directory and configuring and building there.

\subsection{Building in Standalone Mode}

To configure and build ML as a standalone package without any AzTEC support, do the following. It's assumed that the shell variable \$ML_HOME identifies the ML directory.

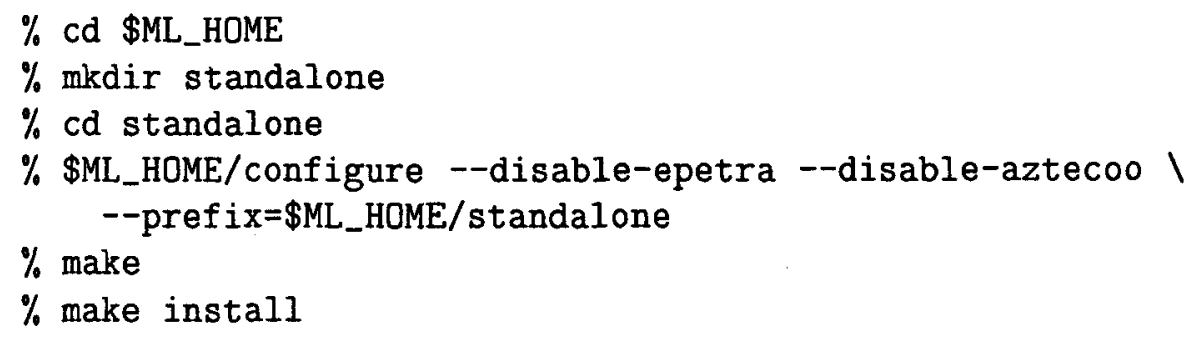

The ML library file libml.a and the header files will be installed in the directory specified in --prefix.

\subsection{Building with AzTEC 2.1 Support}

To enable the supports for AzTEC 2.1, ML must be configured with the options reported in the previous section, plus --with-ml_aztec2_1 (defaulted to no).

All of the AzTEC 2.1 functionality that ML accesses is contained in the file ml_aztec_utils.c. In principal by creating a similar file, other solver packages could work with ML in the same way. For the AzTEC users there are essentially three functions that are important. The first is AZ_ML_Set_Amat which converts AzTEC matrices into ML matrices by making appropriate ML calls (see Section 11.1 and Section 11.2). It is important to note that when creating ML matrices from AzTEC matrices information is not copied. Instead, wrapper functions are made so that ML can access the same information as AzTEC. The second is ML_Gen_SmootherAztec that is used for defining AzTEC iterative methods as smoothers (discussed in Section 8 and Section 13). The third function, AZ_set_ML_preconditioner, can be invoked to set the AzTEC preconditioner to use the multilevel ' $V$ ' cycle constructed in ML. Thus, it is possible to invoke several instances of AzTEC within one solve: smoother on different multigrid levels and/or outer iterative solve.

\subsection{Building with TrILINOS Support (RECOMMENDED)}

We recommend to configure and build ML as part of the standard TRILINos build and configure process. In fact, $\mathrm{ML}$ is built by default if you follow the standard TRILINos configure and build directions. Please refer to the TRILINOS documentation for information about the configuration and building of other TRILINOS packages.

To configure and build ML through TRILINOS, you may need do the following (actual configuration options may vary depending on the specific architecture, installation, and user's need). It's assumed that shell variable \$TRILINOS_HOME identifies the TRILINOS directory, and, for example, that we are compiling under LINUX and MPI. 
$\%$ cd \$TRILINOS_HOME

$\%$ mkdir LINUX_MPI

$\%$ cd LINUX_MPI

$\%$ \$TRILINOS_HOME/configure --with-mpi-compilers ।

--prefix=\$TRILINOS_HOME/LINUX_MPI

$\%$ make

$\%$ make install

If required, other TRILINOS and ML options can be specified in the configure line. A complete list of ML options is given in Section 4.3.1 and 4.3.2. You can also find a complete list and explanations by typing ./configure --help in the ML home directory.

\subsubsection{Enabling Third Party Library Support}

ML can be configured with the following third party libraries (TPLs): SuPERLU, SuPERLU_DIST, METIS, and PARMETIS. It can take advantage of the following TRILINos packages: IfPaCK, Teuchos, Triutils, Amesos. Through Amesos, ML can interface with the direct solvers KLU, UMFPACK, SUPERLU, SUPERLU_DIST ${ }^{3}$, MUMPS. It is assumed that you have already built the appropriate libraries (e.g., libsuperlu.a) and have the header files. To configure ML with one of the above TPLs, you must enable the particular TPL interface in ML. All of the options below are disabled by default.

The same configure options that one uses to enable certain other Trilinos packages also enables the interfaces to those packages within $\mathbf{M L}$ :

--enable-epetra

--enable-aztecoo

--enable-amesos

--enable-teuchos

--enable-triutils

--enable-ifpack
Enable support for the EPETRA package.

Enable support for the AzTECOO package.

Enables support for the AMEsos package. AMEsos is an interface with several direct solvers. ML supports UMFPACK [2], KLU, SUPERLU_dist (1.0 and 2.0), MuMPS [14]. This package is used only in function ML_Gen_SmootherAmesos.

Enables support for the TEuchos package. This package is used only in the definition of class ML_Epetra::MultiLevelPreconditioner (see Section 5). and by the Amesos smoother

Enables support for the TRIUTILS package. ML uses TriutiLs only in some examples, to create the linear system matrix.

Enable support for the IFPACK package [9]. IFPACK is used only to create smoothers via ML_Gen_Smootherlfpack.

\footnotetext{
${ }^{3}$ Currently, ML can support SUPERLU_DIST directly (without AmEsos support), or through AmEsos.
} 

ANASAZI is a high level interface package for various eigenvalue computations.

The following configure line options enable interfaces in ML to certain TPLs.

--with-ml_metis

--with-ml_parmetis $2 x$

--with-ml_parmetis $3 x$

--with-ml_superlu

--with-ml_superlu_dist
Enables interface for METIS [12].

Enables interface for PARMETIS, version 2.x.

Enables interface for PARMETIS [11], version 3.x.

Enables ML interface for serial SUPERLU [3]. The ML interface to SUPERLU is deprecated in favor of the AMESOS interface.

Enables ML interface for SUPERLU_DIST [3]. The ML interface to SUPERLU_DIST is deprecated in favor of the AMESOS interface.

For Metis, ParMETIS, and the ML interface to SuperLU and SUPERLU_dist, the user must specify the location of the header files, with the option

--with-incdirs=include-locations

(Header files for TRILINos libraries are automatically located if ML is built through the TRILINOS configure.) In order to link the ML examples, the user must indicate the location of all the enabled packages' libraries ${ }^{4}$, with the option

--with-ldflags=lib-locations

The user might find useful the option

--disable-examples

which turns off compilation and linking of the examples.

More details about the installation of TRILINOS can be found at the TRILINOS web site, http://software.sandia.gov/Trilinos

and $[10$, Chapter 1].

\subsubsection{Enabling Profiling}

All of the options below are disabled by default.

--enable-ml_timing

This prints out timing of key ML routines.

--enable-ml_flops

This enables printing of flop counts.

Timing and flop counts are printed when the associated object is destroyed.

\footnotetext{
${ }^{4} \mathrm{An}$ example of configuration line that enables METIS and PARMETIS might be as follows: ./configure --with-mpi-compilers --enable-ml_netis --enable-ml_parmetis3x --rith-cflags="-I\$HOME/include" --with-cppflags="-I\$HOME/include" --with-ldflags="-L\$HOME/1ib/LINUX_MPI -1parmetis-3.1 -1metis-4.0" .
} 


\section{ML and Epetra: Getting Started with the MultiLevelPrecon- ditioner Class}

In this Section we show how to use ML as a preconditioner to EPETRA and AzTECOO through the MultiLevelPreconditioner class ${ }^{5}$ in the ML_Epetra namespace. ${ }^{6}$ Although limited to algebraic multilevel preconditioners, this allows the use of ML as a black-box preconditioner.

The MultiLevelPreconditioner class automatically constructs all the components of the preconditioner, using the parameters specified in a TEUCHOS parameter list. The constructor of this class takes as input an Epetra_RowMatrix pointer and a TEUCHOS parameter list?.

In order to compile, it may also be necessary to include the following files: ml_config . $\mathrm{h}$ (as first ML include), Epetra_ConfigDefs.h (as first EPETRA include), Epetra_RowMatrix.h, Epetra_MultiVector.h, Epetra_LinearProblem.h, and Aztec00.h. Check the EPETRA and AzTECOO documentation for more details. Additionally, the user must include the header file "ml_epetra_preconditioner.h". Also note that the macro HAVE_CONFIG_H must be defined either in the user's code or as a compiler flag.

\subsection{Example 1: ml_example_epetra_preconditioner.cpp}

We now give a very simple fragment of code that uses the MultiLevelPreconditioner. For the complete code, see \$ML_HOME/examples/mI_example_epetra_preconditioner.cpp. (In order to be effectively compiled, this example requires ML to be configured with option --enable-triutils; see Section 4.) The linear operator A is derived from an Epetra_RowMatrix, Solver is an AztecOO object, and Problem is an Epetra_LinearProblem object.

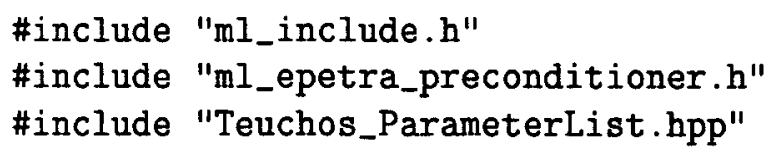

Teuchos : :ParameterList MList;

// set default values for smoothed aggregation in MLList

ML_Epetra: :SetDefaults ("SA", MLList);

$/ /$ overwrite with user's defined parameters

MLList.set ("max levels",6);

MLList.set ("increasing or decreasing", "decreasing");

MLList.set ("aggregation: type", "MIS");

MLList.set ("coarse: type", "Amesos-KLU");

\footnotetext{
${ }^{5}$ The MultiLevelPreconditioner class is derived from the Epetra.RowMatrix class.

${ }^{6} \mathrm{ML}$ does not rely on any particular matrix format or iterative solver. Examples of using of ML as a preconditioner for user-defined matrices (i.e., non-Epetra matrices) are reported in Section 11.1 and 11.2.

${ }^{7}$ In order to use the MultiLevelPreconditioner class, ML must be configured with options -enable-epetra --enable-teuchos.
} 


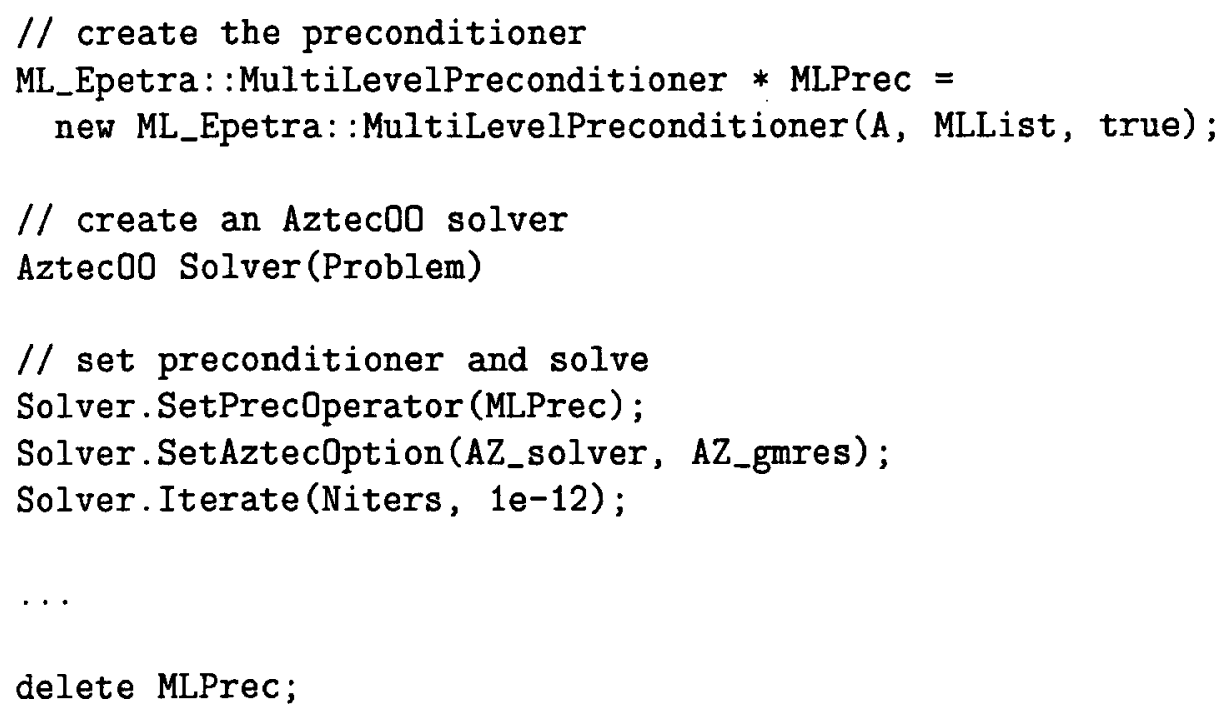

We now detail the general procedure to define the MultiLevelPreconditioner. First, the user defines a TEuCHOS parameter list ${ }^{8}$. Table 2 briefly reports the most important methods of this class.

\begin{tabular}{|l|l|}
\hline set (Name, Value) & Add entry Name with value and type specified by Value. Any \\
& C++ type (like int, double, a pointer, etc.) is valid. \\
get (Name,DefValue) & Get value (whose type is automatically specified by DefValue). If \\
& not present, return DefValue. \\
subList (Name) & Get a reference to sublist List. If not present, create the sublist. \\
\hline
\end{tabular}

Table 2: Some methods of Teuchos::ParameterList class.

Input parameters are set via method set (Name, Value), where Name is a string defining the parameter, and Value is the specified parameter, that can be any $\mathrm{C}++$ object or pointer. A complete list of parameters available for class MultiLevelPreconditioner is reported in Section 6.

The parameter list is passed to the constructor, together with a pointer to the matrix, and a boolean flag. If this flag is set to false, the constructor will not create the multilevel hierarchy until when MLPrec->ComputePreconditioner() is called. The hierarchy can be destroyed using MLPrec->Destroy ()$^{9}$. For instance, the user may define a code like:

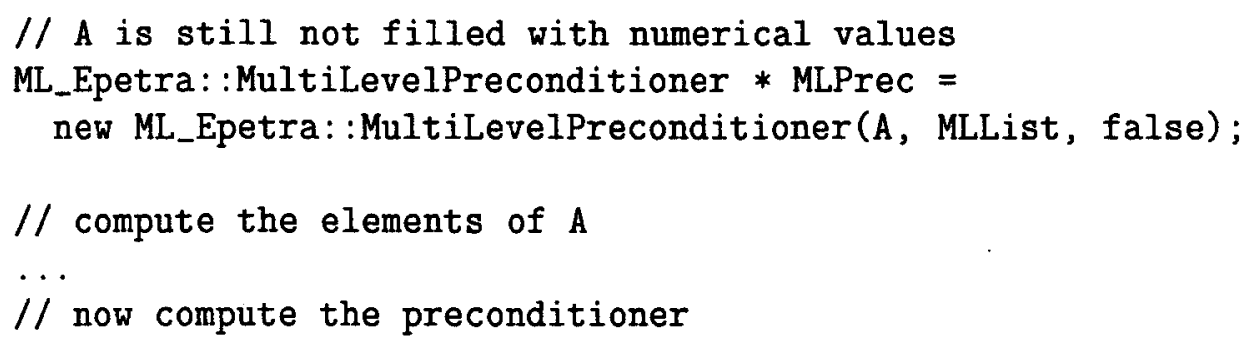

\footnotetext{
${ }^{8}$ See the TEuchos documentation for a detailed overview of this class.

${ }^{9}$ We suggest to create the preconditioning object with neu and to free memory with delete. Some MPI calls occur in Destroy(), so the user should not call MPI_Finalize() or delete the communicator used by ML before the preconditioning object is destroyed.
} 
MLPrec->ComputePreconditioner();

// solve the linear system

...

// destroy the previously define preconditioner, and build a new one MLPrec->Destroy();

// re-compute the elements of $\mathrm{A}$

$/ /$ now re-compute (if required) the preconditioner

MLPrec->ComputePreconditioner();

\section{// re-solve the linear system}

In this fragment of code, the user defines the ML preconditioner, but the preconditioner is created only with the call ComputePreconditioner (). This may be useful, for example, when $\mathrm{ML}$ is used in conjunction with nonlinear solvers (like Nox [13]).

\subsection{Example 2: ml_example_epetra_preconditioner_2level.cpp}

As a second example, here we explain with some details the construction of a 2-level domain decomposition preconditioner, with a coarse space defined using aggregation.

File \$ML_HOME/examples/ml_example_epetra_preconditioner_2level.cpp reports the entire code. In the example, the linear system matrix A, coded as an Epetra_CrsMatrix, corresponds to the discretization of a $2 \mathrm{D}$ Laplacian on a Cartesian grid. $\mathrm{x}$ and $\mathrm{b}$ are the solution vector and the right-hand side, respectively.

The AztecOO linear problem is defined as

Epetra_LinearProblem problem(\&A, \&x, \&b);

Azteco0 solver (problem);

We create the TEUCHOS parameter list as follows:

ParameterList MLList;

ML_Epetra: :SetDefaults("DD", MLList);

MLList.set ("max levels",2);

MLList.set("increasing or decreasing", "increasing");

MLList.set("aggregation: type", "METIS");

MLList.set("aggregation: nodes per aggregate", 16);

MLList.set ("smoother: pre or post", "both");

MLList.set ("coarse: type", "Amesos-KLU");

MLList.set("smoother: type", "Aztec");

The last option tells ML to use the AzTEC preconditioning function as a smoother. All AzTEC preconditioning options can be used as ML smoothers. AzTEC requires an integer vector options and a double vector params. Those can be defined as follows:

int options[AZ_OPTIONS_SIZE];

double params [AZ_PARAMS_SIZE]; 


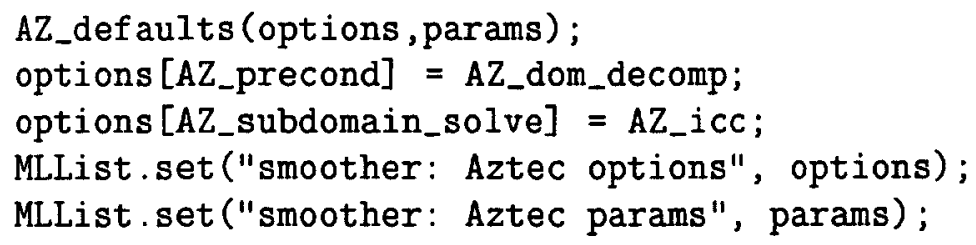

The last two commands set the pointer to options and params in the parameter list ${ }^{10}$.

The ML preconditioner is created as in the previous example,

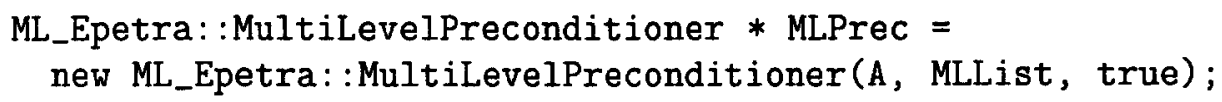

and we can check that no options have been mispelled, using

MLPrec->PrintUnused ();

The AztecOO solver is called using, for instance,

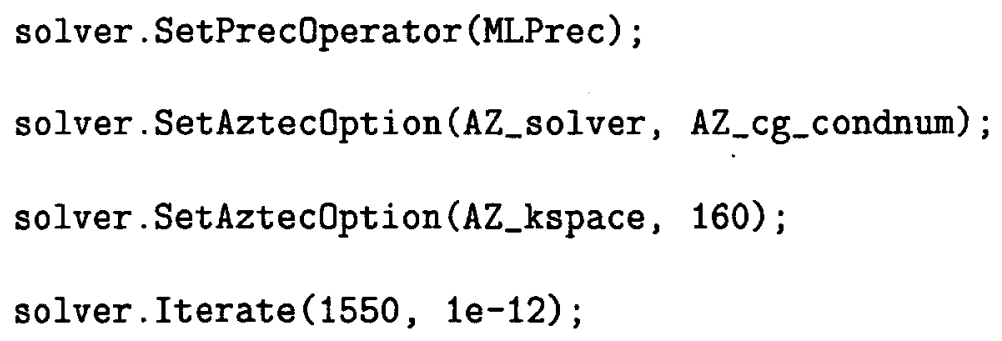

Finally, some (limited) information about the preconditioning phase are obtained using cout $\ll$ MLPrec->GetOutputList();

Note that the input parameter list is copied in the construction phase, hence later changes to MLList will not affect the preconditioner. Should the user need to modify parameters in the MLPrec's internally stored parameter list, he can get a reference to the internally stored list:

ParameterList \& List $=$ MLPrec $->$ GetList () ;

and then directly modify List.

\section{Parameters for the ML_Epetra::MultiLevelPreconditioner Class}

In this section we give general guidelines for using the MultiLevelPreconditioner class effectively. The complete list of input parameters is also reported.

\footnotetext{
${ }^{10}$ Only the pointer is copied in the parameter list, not the array itself. Therefore, options and params should not go out of scope before the destruction of the preconditioner.
} 


\subsection{Setting Options on a Specific Level}

Some of the parameters that affect MultiLevelPreconditioner can in principle be different from level to level. By default, the set method for the MultiLevelPreconditioner class affects all levels in the multigrid hierarchy. In order to change a setting on a particular level (say, d), the string "(level d)" is appended to the option string (note that a space must separate the option and the level specification). For instance, assuming decreasing levels starting from 4 , one could set the aggregation schemes as follows:

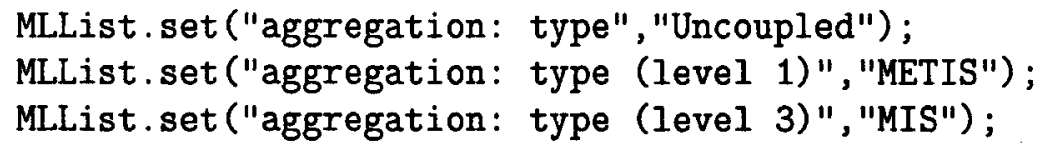

If the finest level is 0 , and one has 5 levels, the code will use Uncoupled for level 0, METIS for levels 1 and 2, then MIS for levels 3 and 4.

In $\S 6.5$, parameters that can be set differently on individual levels are denoted with the symbol $\star$ (that is not part of the parameter name). Note that some parameters (e.g., Uncoupled-MIS aggregation) correspond to quantities that must be the same at all levels.

\subsection{General Usage of the Parameter List}

All ML options can have a common prefix, specified by the user in the construction phase. For example, suppose that we require ML: (in this case with a trailing space) to be the prefix. The constructor will be

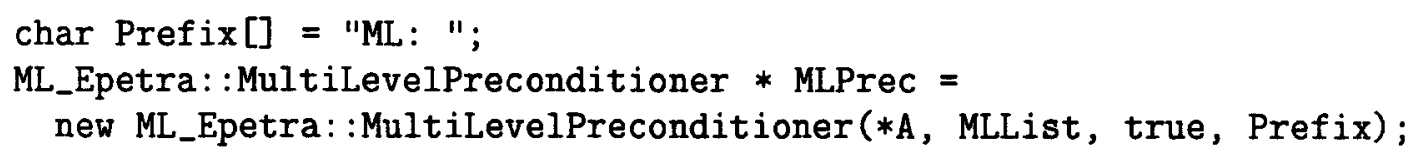

A generic parameter, say aggregation: type, will now be defined as

MLLIst.set("ML: aggregation: type", "METIS");

It is important to point out that some options can be effectively used only if ML has been properly configured. In particular:

- METIS aggregation scheme requires --with-ml_metis, or otherwise the code will include all nodes in the calling processor in a unique aggregate;

- PARMetis aggregation scheme required --with-ml_metis --enable-epetra and --with-ml_parmetis $2 x$ or --with-ml_parmetis $3 x$.

- AmEsos coarse solvers require --enable-amesos. Moreover, AmEsos must have been configure to support the requested coarse solver. Please refer to the AMEsos documentation for more details;

- IFPACK smoother requires --enable-ifpack. 


\subsection{Default Parameter Settings for Common Problem Types}

The MultiLevelPreconditioner class provides default values for four different preconditioner types:

1. Linear elasticity

2. Classical 2-level domain decomposition for the advection diffusion operator

3. 3-level algebraic domain decomposition for the advection diffusion operator

4. Eddy current formulation of Maxwell's equations

Default values are listed in Table 3. In the table, SA refers to "classical" smoothed aggregation (with small aggregates and relative large number of levels), DD and DD-ML to domain decomposition methods (whose coarse matrix is defined using aggressive coarsening and limited number of levels). Maxwell refers to the solution of Maxwell's equations.

Default values for the parameter list can be set by ML_Epetra: :SetDefaults(). The user can easily put the desired default values in a given parameter list as follows:

Teuchos: : ParameterList MLList;

ML_Epetra: :SetDefaults (ProblemType, MLList);

or as

Teuchos: : ParameterList MLList;

ML_Epetra: :SetDefaults(ProblemType, MLList, Prefix);

Prefix (defaulted to an empty string) is the prefix to assign to each entry in the parameter list.

For DD and DD-ML, the default smoother is Aztec, with an incomplete factorization ILUT, and minimal overlap. Memory for the two AzTEC vectors is allocated using new, and the user is responsible to free this memory, for instance as follows:

int * options;

options = MLList.get ("smoother: Aztec options", options);

double * params;

params $=$ MLList.get ("smoother: Aztec params", params);

.

// Make sure solve is completed before deleting options \& params!!

delete [] options;

delete [] params;

The rational behind this is that the parameter list stores a pointer to those vectors, not the content itself. (As a general rule, the vectors stored in the parameter list should not be prematurely destroyed or permitted to go out of scope.) 


\begin{tabular}{|c|c|c|c|c|c|}
\hline Option Name & Type & SA & $\mathrm{DD}$ & DD-ML & maxwe11 \\
\hline $\begin{array}{l}\text { max levels } \\
\text { output } \\
\text { increasing or decreasing } \\
\text { PDE equations } \\
\text { null space dimension } \\
\text { null space vectors }\end{array}$ & $\begin{array}{l}\text { int } \\
\text { int } \\
\text { string } \\
\text { int } \\
\text { int } \\
\text { double* }\end{array}$ & $\begin{array}{l}16 \\
8 \\
\text { increasing } \\
1 \\
1 \\
\text { NULL } \\
\end{array}$ & $\begin{array}{l}2 \\
8 \\
\text { increasing } \\
1 \\
1 \\
\text { NULL }\end{array}$ & $\begin{array}{l}3 \\
8 \\
\text { increasing } \\
1 \\
1 \\
\text { NULL }\end{array}$ & $\begin{array}{l}5 \\
10 \\
\text { decreasing } \\
- \\
- \\
\text { NULL }\end{array}$ \\
\hline $\begin{array}{l}\text { aggregation: type } \\
\text { aggregation: type (level 1) } \\
\text { aggregation: type (level 8) } \\
\text { aggregation: local aggregates } \\
\text { aggregation: nodes per aggregate } \\
\text { aggregation: damping factor } \\
\text { eigen-analysis: type } \\
\text { coarse: max size } \\
\text { aggregation: threshold } \\
\text { aggregation: next-level aggregates } \\
\text { per process }\end{array}$ & $\begin{array}{l}\text { string } \\
\text { string } \\
\text { string } \\
\text { int } \\
\text { int } \\
\text { double } \\
\text { string } \\
\text { int } \\
\text { double } \\
\text { int }\end{array}$ & $\begin{array}{l}\text { Uncoupled } \\
- \\
\text { MIS } \\
- \\
- \\
4 / 3 \\
\text { Anorm } \\
128 \\
0.0 \\
-\end{array}$ & $\begin{array}{l}\text { METIS } \\
- \\
- \\
1 \\
- \\
4 / 3 \\
\text { Anorm } \\
128 \\
0.0 \\
-\end{array}$ & $\begin{array}{l}\text { METIS } \\
\text { ParMETIS } \\
- \\
- \\
512 \\
4 / 3 \\
\text { Anorm } \\
128 \\
0.0 \\
128\end{array}$ & $\begin{array}{l}\text { Uncoupled-MIS } \\
- \\
- \\
- \\
- \\
0.0 \\
\text { Anorm } \\
128 \\
0.0 \\
-\end{array}$ \\
\hline $\begin{array}{l}\text { smoother: } \\
\text { sweeps } \\
\text { smoother: damping factor } \\
\text { smoother: type } \\
\text { smoother: Aztec as solver } \\
\text { smoother: MLS polynomial order } \\
\text { smoother: MLS alpha }\end{array}$ & $\begin{array}{l}\text { int } \\
\text { double } \\
\text { string } \\
\text { string } \\
\text { bool } \\
\text { int } \\
\text { double }\end{array}$ & $\begin{array}{l}2 \\
0.67 \\
\text { both } \\
\text { Gauss-Seidel } \\
- \\
- \\
- \\
\end{array}$ & $\begin{array}{l}2 \\
- \\
\text { both } \\
\text { Aztec } \\
\text { false } \\
- \\
-\end{array}$ & $\begin{array}{l}2 \\
- \\
\text { both } \\
\text { Aztec } \\
\text { false } \\
- \\
-\end{array}$ & $\begin{array}{l}2 \\
0.67 \\
\text { both } \\
- \\
- \\
3 \\
30.0 \\
\end{array}$ \\
\hline $\begin{array}{l}\text { coarse: type } \\
\text { coarse: sweeps } \\
\text { coarse: damping factor } \\
\text { coarse: max processes } \\
\text { print unused }\end{array}$ & $\begin{array}{l}\text { string } \\
\text { int } \\
\text { double } \\
\text { int } \\
\text {-int } \\
\end{array}$ & $\begin{array}{l}\text { Amesos.KLU } \\
1 \\
1.0 \\
16 \\
0 \\
\end{array}$ & $\begin{array}{l}\text { Amesos KLU } \\
1 \\
1.0 \\
16 \\
0\end{array}$ & $\begin{array}{l}\text { Amesos KLU } \\
1 \\
1.0 \\
16 \\
0\end{array}$ & $\begin{array}{l}\text { SuperLU } \\
1 \\
1.0 \\
- \\
0\end{array}$ \\
\hline
\end{tabular}

Table 3: Default values for ML_Epetra::MultiLevelPreconditioner for the 4 currently supported problem types SA, DD, DD-ML, Maxwell. "_" means not set. 


\begin{tabular}{|l|l|}
\hline Uncoupled & $\begin{array}{l}\text { Attempts to construct aggregates of optimal size (3 } 3^{d} \text { nodes in } d \\
\text { dimensions). Each process works independently, and aggregates } \\
\text { cannot span processes. }\end{array}$ \\
MIS & $\begin{array}{l}\text { As Uncoupled, but aggregates can span processes (deprecated). } \\
\text { Uses a maximal independent set technique to define the aggre- } \\
\text { gates. Aggregates can span processes. May provide better qual- } \\
\text { ity aggregates than either Coupled or uncoupled. Computation- } \\
\text { ally more expensive than either because it requires matrix-matrix } \\
\text { product. } \\
\text { Uncoupled-MIS } \\
\text { Uses Uncoupled for all levels until there is 1 aggregate per pro- } \\
\text { cessor. Then switches over to MIS. The coarsening scheme on a } \\
\text { given level cannot be specified with this option. } \\
\text { Use a graph partitioning algorithm to creates the aggregates, } \\
\text { working process-wise. The number of nodes in each aggregate is } \\
\text { specified with the option aggregation: nodes per aggregate. } \\
\text { Requires ML to be configured with --with-ml_metis. } \\
\text { As METIS, but partition the global graph. } \\
\text {--with-ml-parmetis2x or -with-ml-parmetis3x. Aggregates } \\
\text { can span arbitrary number of processes. Global number of ag- } \\
\text { gregates can be specified with the option aggregation: global } \\
\text { number. }\end{array}$ \\
\hline
\end{tabular}

Table 4: ML_Epetra::MultiLevelPreconditioner: Available coarsening schemes.

\subsection{Commonly Used Parameters}

Table 4 lists parameter for changing aggregation schemes. Table 5 lists common choices for smoothing options. Table 6 lists common choices affecting the coarse grid solve.

Note that, in the parameters name, spaces are important: Do not include nonrequired leading or trailing spaces, and separate words by just one space! Mispelled parameters will not be detected. One may find useful to print unused parameters by calling PrintUnused() after the construction of the multilevel hierarchy.

\subsection{List of All Parameters for MultiLevelPreconditioner Class}

\subsubsection{General Options}

output

print unused

$\max$ levels

increasing or decreasing
Output level, from 0 to 10 (10 being verbose).

If non-negative, will print all the unused parameter on the specified processor.

Maximum number of levels.

If set to increasing, level 0 will correspond to the finest level. If set to decreasing, max levels - 1 will correspond to the finest level. 


\begin{tabular}{|l|l|}
\hline Jacobi & $\begin{array}{l}\text { Point-Jacobi. Damping factor is specified using smoother: } \\
\text { dampig factor, and the number of sweeps with smoother: } \\
\text { sweeps } \\
\text { Point Gauss-Seidel. Damping factor is specified using smoother: } \\
\text { dampig factor, and the number of sweeps with smoother: } \\
\text { sweeps } \\
\text { Use AzTECOO's built-in preconditioning functions as smoothers. } \\
\text { Or, if smoother: Aztec as solver is true, use approximate } \\
\text { solutions with AzTECOO(with smoothers: sweeps iterations } \\
\text { as smoothers. The AzTECOOvectors options and params can } \\
\text { be set using smoother: Aztec options and smoother: Aztec } \\
\text { params. } \\
\text { Use MLS smoother. The polynomial order is specified by } \\
\text { smoother: MLS polynomial order, and the alpha value by } \\
\text { smoother: MLS alpha. }\end{array}$ \\
\hline
\end{tabular}

Table 5: ML_Epetra::MultiLevelPreconditioner: Commonly used smoothers.

\begin{tabular}{|l|l|}
\hline Jacobi & $\begin{array}{l}\text { Use coarse: sweeps steps of Jacobi (with damping parameter } \\
\text { coarse: damping parameter) as a solver. } \\
\text { Use coarse: sweeps steps of Gauss-Seidel(with damping pa- } \\
\text { rameter coarse: damping parameter) as a solver. }\end{array}$ \\
Ase KLUthrough AMESOS. Coarse grid problem is shipped to proc \\
Use-KLU & $\begin{array}{l}\text { 0, solved, and solution is broadcast } \\
\text { Use UMFPACK through AMESOS. Coarse grid problem is shipped } \\
\text { to proc 0, solved, and solution is broadcasted. }\end{array}$ \\
Amesos-UMFPACK & Use SUPERLU_DISTthrough AMESOS. \\
Amesos-MUMPS & Use double precision version of MUMPS through AMESOS. \\
Amesos-ScaLAPACK & Use double precision version of SCALAPACK through AMESOS. \\
SuperLU & Use ML interface to SUPERLU. \\
\hline
\end{tabular}

Table 6: ML_Epetra::MultiLevelPreconditioner: Some of the available coarse matrix solvers. Note: Amesos solvers requires ML to be configured with with-ml_amesos, and Amesos to be properly configured to support the specified solver.

PDE equations

null space dimension

null space vectors
Number of PDE equations for each grid node. This value is not considered for Epetra_VbrMatrix objects, as in this case is obtained from the block map used to construct the object. Note that only block maps with constant element size can be considered.

Dimension of the null space.

Pointer to the null space vectors. If NULL, $\mathbf{M L}$ will use the default null space. 


\subsubsection{Aggregation Parameters}

aggregation: type $\star$

aggregation: global aggregates $\star$

aggregation: local aggregates *

aggregation: nodes per aggregate $\star$ Defines the number of nodes to be assigned to each aggregate (only for METIS and ParMETIS aggregation schemes). Note: this value overwrites aggregation: local aggregates. If none among aggregation: global aggregates, aggregation: local aggregates and aggregation: nodes per aggregate is specified, the default value is 1 aggregate per process.

aggregation: damping factor

eigen-analysis: type

aggregation: threshold

aggregation: next-level aggregates Defines the maximum number of next-level maper process *

\subsubsection{Smoothing Parameters}

smoother: sweeps $\star \quad$ Number of sweeps of smoother.
Damping factor for smoothed aggregation.

Defines the numerical scheme to be used to compute an estimation of the maximum eigenvalue of $D^{-1} A$, where $D=\operatorname{diag}(A)$ (for smoothed aggregation only). It can be: cg (use 10 steps of conjugate gradient method), Anorm (use Anorm of matrix), Anasazi (use the ANASAzI package; the problem is supposed to be nonsymmetric), or power-method.

Threshold in aggregation. trix rows per process (only for ParMETIS aggregation scheme). 
smoother: damping factor *

smoother: pre or post $\star$

smoother: type *

smoother: Aztec options *

smoother: Aztec params $\star$

smoother: Aztec as solver *
Smoother damping factor.

If set to pre, only pre-smoothing will be used. If set to post, only post-smoothing will be used. If set to both, pre- and post-smoothing will be used.

Type of the smoother. It can be: Jacobi, Gauss-Seidel, sym Gauss-Seidel, Aztec, IFPACK. See Table 5.

Pointer to AzTEC's options vector (only for aztec smoother) .

Pointer to AzTEC's params vector (only for aztec smoother) .

If true, smoother: sweeps iterations of AzTEC solvers will be used as smoothers. If false, only the AzTEC's preconditioner function will be used as smoother (only for aztec smoother) .

smoother: MLS polynomial order $\star$ Polynomial order for MLS smoothers.

smoother: MLS alpha *

Alpha value for MLS smoothers.

\subsubsection{Coarsest Grid Parameters}

coarse: $\max$ size

Maximum dimension of the coarse grid. ML will not coarsen further is the size of the current level is less than this value.

coarse: type

coarse: sweeps

coarse: damping factor
Coarse solver. It can be: Jacobi, Gauss-Seidel, Amesos_KLU, Amesos_UMFPACK, Amesos_Superludist, Amesos_MUMPS. See Table 6.

(only for Jacobi and Gauss-Seidel) Number of sweeps in the coarse solver.

(only for Jacobi and Gauss-Seidel) Damping factor in the coarse solver. 
coarse: max processes
Maximum number of processes to be used in the coarse grid solution (only for Amesos-Superludist, Amesos-MUMPS, Amesos-ScaLAPACK).

\section{Advanced Usage of ML}

Sections 5 and 6 have detailed the use of ML as a black box preconditioner. In some cases, instead, the user may need to explicitly construct the ML hierarchy. This is reported in the following sections.

A brief sample program is given in Figure 2. The function ML_Create creates a mul-

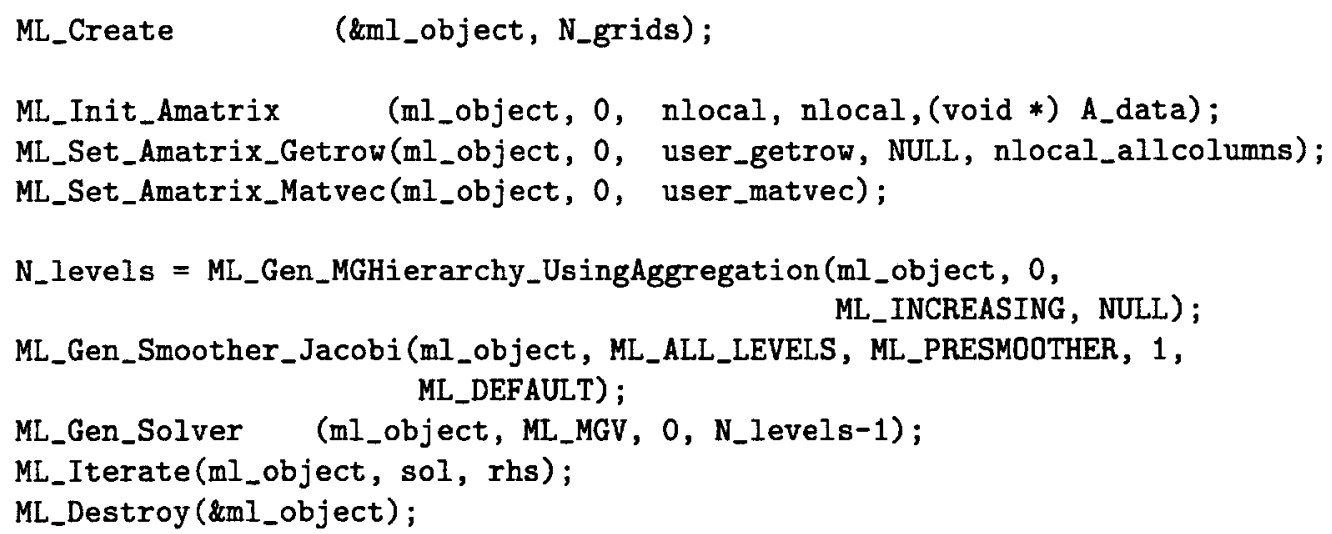

Figure 2: High level multigrid sample code.

tilevel solver object that is used to define the preconditioner. It requires the maximum number of multigrid levels be specified. In almost all cases, N_grids $=20$ is more than adequate. The three 'Amatrix' statements are used to define the discretization matrix, $A$, that is solved. This is discussed in greater detail in Section 11.1. The multigrid hierarchy is generated via ML_Gen_MGHierarchy_UsingAggregation. Controlling the behavior of this function is discussed in Section 9. For now, it is important to understand that this function takes the matrix $A$ and sets up relevant multigrid operators corresponding to the smoothed aggregation multigrid method [18] [17]. In particular, it generates a graph associated with $A$, coarsens this graph, builds functions to transfer vector data between the original graph and the coarsened graph, and then builds an approximation to $A$ on the coarser graph. Once this second multigrid level is completed, the same operations are repeated to the second level approximation to $A$ generating a third level. This process continues until the current graph is sufficiently coarse. The function ML_Gen_Smoother_Jacobi indicates that a Jacobi smoother should be used on all levels. Smoothers are discussed further in Section 8. Finally, ML_Gen_Solver is invoked when the multigrid preconditioner is fully specified. This function performs any needed initialization and checks for inconsistent options. After ML_Gen_Solver completes ML_Iterate can be used to solve the problem with an initial guess 
of sol (which will be overwritten with the solution) and a right hand side of rhs. At the present time, the external interface to vectors are just arrays. That is, rhs and sol are simple one-dimensional arrays of the same length as the number of rows in $A$. In addition to ML_Iterate, the function ML_Solve_MGV can be used to perform one multigrid ' $V$ ' cycle as a preconditioner.

\section{Multigrid \& Smoothing Options}

Several options can be set to tune the multigrid behavior. In this section, smoothing and high level multigrid choices are discussed. In the next section, the more specialized topic of the grid transfer operator is considered. The details of the functions described in these next two sections are given in Section 13.

For most applications, smoothing choices are important to the overall performance of the multigrid method. Unfortunately, there is no simple advice as to what smoother will be best and systematic experimentation is often necessary. ML offers a variety of standard smoothers. Additionally, user-defined smoothers can be supplied and it is possible to use AzTECas a smoother. A list of ML functions that can be invoked to use built-in smoothers are given below along with a few general comments.

ML_Gen_Smoother_Jacobi

ML_Gen_Smoother_GaussSeidel

ML_Gen_Smoother_SymGaussSeidel

ML_Gen_Smoother_BlockGaussSeidel

ML_Gen_Smoother_VBlockJacobi
Typically, not the fastest smoother. Should be used with damping. For Poisson problems, the recommended damping values are $\frac{2}{3}$ (1D), $\frac{4}{5}$ (2D), and $\frac{5}{7}(3 \mathrm{D})$. In general, smaller damping numbers are more conservative.

Probably the most popular smoother. Typically, faster than Jacobi and damping is often not necessary nor advantageous.

Symmetric version of Gauss Seidel. When using multigrid preconditioned conjugate gradient, the multigrid operator must be symmetrizable. This can be achieved by using a symmetric smoother with the same number of pre and post sweeps on each level.

Block Gauss-Seidel with a fixed block size. Often used for PDE systems where the block size is the number of degrees of freedom (DOFs) per grid point.

Variable block Jacobi smoother. This allows users to specify unknowns to be grouped into different blocks when doing block Jacobi. 
ML_Gen_Smoother_VBlockSymGaussSeidel Symmetric variable block Gauss-Seidel smoothing. This allows users to specify unknowns to be grouped into different blocks when doing symmetric block Gauss-Seidel.

It should be noted that the parallel Gauss-Seidel smoothers are not true Gauss-Seidel. In particular, each processor does a Gauss-Seidel iteration using off-processor information from the previous iteration.

AzTEC user's [15] can invoke ML_Gen_SmootherAztec to use either AzTEC solvers or AzTEC preconditioners as smoothers on any grid level. Thus, for example, it is possible to use preconditioned conjugate-gradient (where the preconditioner might be an incomplete Cholesky factorization) as a smoother within the multigrid method. Using Krylov smoothers as a preconditioner could potentially be more robust than using the simpler schemes provided directly by ML. However, one must be careful when multigrid is a preconditioner to an outer Krylov iteration. Embedding an inner Krylov method within a preconditioner to an outer Krylov method may not converge due to the fact that the preconditioner can no longer be represented by a simple matrix. Finally, it is possible to pass user-defined smoothing functions into ML via ML_Set_Smoother. The signature of the user defined smoother function is

int user_smoothing(void *data, int $x_{-}$length, double $x[]$, int rhs_length, double rhs[])

where data is a pointer given with the ML_Set_Smoother invocation, $\mathrm{x}$ is a vector (of length $\mathrm{x}$-length) that corresponds to the initial guess on input and is the improved solution estimate on output, and rhs is the right hand side vector of length rhs_length. A simple (and suboptimal) damped Jacobi smoother for the finest grid of our example is given below:

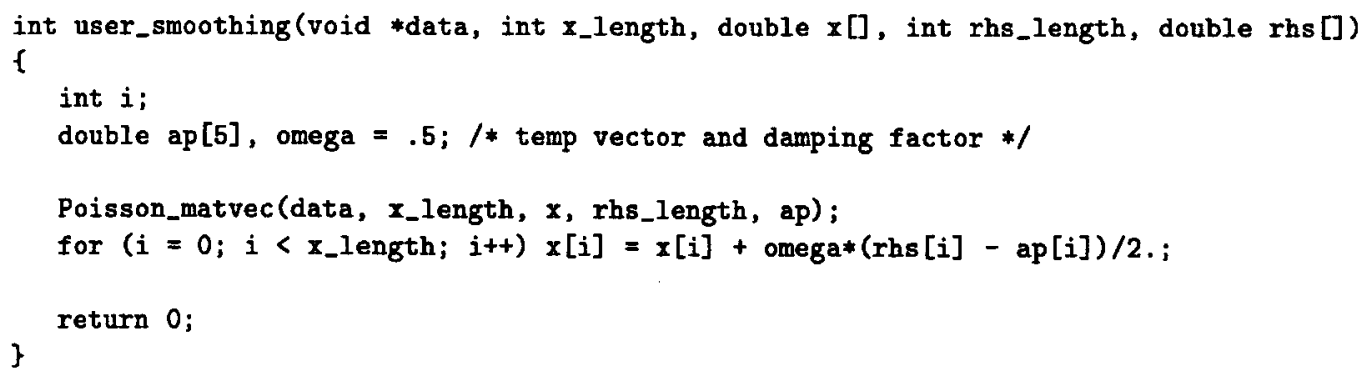

A more complete smoothing example that operates on all multigrid levels is given in the file mlguide.c. This routine uses the functions ML_Operator_Apply, ML_Operator_Get_Diag, and ML_Get_Amatrix to access coarse grid matrices constructed during the algebraic multigrid process. By writing these user-defined smoothers, it is possible to tailor smoothers to a particular application or to use methods provided by other packages. In fact, the AzTEC methods within ML have been implemented by writing wrappers to existing AzTEC functions and passing them into ML via ML_Set_Smoother.

At the present time there are only a few supported general parameters that may be altered by users. However, we expect that this list will grow in the future. When using ML.Iterate, the convergence tolerance (ML_Set_Tolerance) and the frequency with which 
residual information is output (ML_Set_ResidualOutputFrequency) can both be set. Additionally, the level of diagnostic output from either ML_Iterate or ML_Solve_MGV can be set via ML_Set_OutputLevel. The maximum number of multigrid levels can be set via ML_Create or ML_Set_MaxLevels. Otherwise, ML continues coarsening until the coarsest grid is less than or equal to a specified size (by default 10 degrees of freedom). This size can be set via ML_Aggregate_Set_MaxCoarseSize.

\section{Smoothed Aggregation Options}

When performing smooth aggregation, the matrix graph is first coarsened (actually vertices are aggregated together) and then a grid transfer operator is constructed. A number of parameters can be altered to change the behavior of these phases.

\subsection{Aggregation Options}

A graph of the matrix is usually constructed by associating a vertex with each equation and adding an edge between two vertices $i$ and $j$ if there is a nonzero in the $(i, j)^{\text {th }}$ or $(j, i)^{\text {th }}$ entry. It is this matrix graph whose vertices are aggregated together that effectively determines the next coarser mesh. The above graph generation procedure can be altered in two ways. First, a block matrix graph can be constructed instead of a point matrix graph. In particular, all the degrees of freedom (DOFs) at a grid point can be collapsed into a single vertex of the matrix graph. This situation arises when a PDE system is being solved where each grid point has the same number of DOFs. The resulting block matrix graph is significantly smaller than the point matrix graph and by aggregating the block matrix graph, all unknowns at a grid point are kept together. This usually results in better convergence rates (and the coarsening is actually less expensive to compute). To indicate the number of DOFs per node, the function ML_Aggregate_Set_NullSpace is used. The second way in which the graph matrix can be altered is by ignoring small values. In particular, it is often preferential to ignore weak coupling during coarsening. The error between weakly coupled points is generally hard to smooth and so it is best not to coarsen in this direction. For example, when applying a Gauss-Seidel smoother to a standard discretization of

$$
u_{x x}+\epsilon u_{y y}=f
$$

(with $0 \leq \epsilon \leq 10^{-6}$ ), there is almost no coupling in the $y$ direction. Consequently, simple smoothers like Gauss-Seidel do not effectively smooth the error in this direction. If we apply a standard coarsening algorithm, convergence rates suffer due to this lack of $y$-direction smoothing. There are two principal ways to fix this: use a more sophisticated smoother or coarsen the graph only in the $x$ direction. By ignoring the $y$-direction coupling in the matrix graph, the aggregation phase effectively coarsens in only the $x$-direction (the direction for which the errors are smooth) yielding significantly better multigrid convergence rates. In general, a drop tolerance, $t_{d}$, can be set such that an individual matrix entry, $A(i, j)$ is dropped in the coarsening phase if

$$
|A(i, j)| \leq t_{d} * \sqrt{|A(i, i) A(j, j)|} .
$$

This drop tolerance (whose default value is zero) is set by ML_Aggregate_Set_Threshold.

There are two different groups of graph coarsening algorithms in ML: 
- schemes with fixed ratio of coarsening between levels: uncoupled aggregation, coupled aggregation, and MIS aggregation. A description of those three schemes along with some numerical results are given in [16]. As the default, the Uncoupled-MIS scheme is used which does uncoupled aggregation on finer grids and switches to the more expensive MIS aggregation on coarser grids;

- schemes with variable ratio of coarsening between levels: METIS and PARMETISaggregation. Those schemes use the graph decomposition algorithms provided by METIS and PARMETIS, to create the aggregates.

Poorly done aggregation can adversely affect the multigrid convergence and the time per iteration. In particular, if the scheme coarsens too rapidly multigrid convergence may suffer. However, if coarsening is too slow, the number of multigrid levels increases and the number of nonzeros per row in the coarse grid discretization matrix may grow rapidly. We refer the reader to the above paper and indicate that users might try experimenting with the different schemes via ML_Aggregate_Set_CoarsenScheme_Uncoupled, ML_Aggregate_Set_CoarsenScheme_Coupled, ML_Aggregate_Set_CoarsenScheme_MIS, ML_Aggregate_Set_CoarsenScheme_METIS, and ML_Aggregate_Set_CoarsenScheme_ParMETIS.

\subsection{Interpolation Options}

An interpolation operator is built using coarsening information, seed vectors, and a damping factor. We refer the reader to [17] for details on the algorithm and the theory. In this section, we explain a few essential features to help users direct the interpolation process.

Coarsening or aggregation information is first used to create a tentative interpolation operator. This process takes a seed vector or seed vectors and builds a grid transfer operator. The details of this process are not discussed in this document. It is, however, important to understand that only a few seed vectors are needed (often but not always equal to the number of DOFs at each grid point) and that these seed vectors should correspond to components that are difficult to smooth. The tentative interpolation that results from these seed vectors will interpolate the seed vectors perfectly. It does this by ensuring that all seed vectors are in the range of the interpolation operator. This means that each seed vector can be recovered by interpolating the appropriate coarse grid vector. The general idea of smoothed aggregation (actually all multigrid methods) is that errors not eliminated by the smoother must be removed by the coarse grid solution process. If the error after several smoothing iterations was known, it would be possible to pick this error vector as the seed vector. However, since this is not the case, we look at vectors associated with small eigenvalues (or singular values in the nonsymmetric case) of the discretization operator. Errors in the direction of these eigenvectors are typically difficult to smooth as they appear much smaller in the residual $(r=A e$ where $r$ is the residual, $A$ is discretization matrix, and $e$ is the error). For most scalar PDEs, a single seed vector is sufficient and so we seek some approximation to the eigenvector associated with the lowest eigenvalue. It is well known that a scalar Poisson operator with Neumann boundary conditions is singular and that the null space is the constant vector. Thus, when applying smoothed aggregation to Poisson operators, it is quite natural to choose the constant vector as the seed vector. In many cases, this constant vector is a good choice as all spatial derivatives within the operator are zero and so it is often associated with small singular values. Within ML the default is to choose 
the number of seed vectors to be equal to the number of DOFs at each node (given via ML_Aggregate_Set_NullSpace). Each seed vector corresponds to a constant vector for that DOF component. Specifically, if we have a PDE system with two DOFs per node. Then one seed vector is one at the first DOF and zero at the other DOF throughout the graph. The second seed vector is zero at the first DOF and one at the other DOF throughout the graph. In some cases, however, information is known as to what components will be difficult for the smoother or what null space is associated with an operator. In elasticity, for example, it is well known that a floating structure has six rigid body modes (three translational vectors and three rotation vectors) that correspond to the null space of the operator. In this case, the logical choice is to take these six vectors as the seed vectors in smoothed aggregation. When this type of information is known, it should be given to ML via the command ML_Aggregate_Set_NullSpace.

Once the tentative prolongator is created, it is smoothed via a damped Jacobi iteration. The reasons for this smoothing are related to the theory where the interpolation basis functions must have a certain degree of smoothness (see [17]). However, the smoothing stage can be omitted by setting the damping to zero using the function ML_Aggregate_Set_DampingFactor. Though theoretically poorer, unsmoothed aggregation can have considerably less set up time and less cost per iteration than smoothed aggregation. When smoothing, ML has two ways to determine the Jacobi damping parameter and each require some estimate of the largest eigenvalue of the discretization operator. The current default is to use a few iterations of a conjugate-gradient method to estimate this value. However, if the matrix is nonsymmetric, the infinity norm of the matrix should be used instead via ML_Aggregate_Set_SpectralNormScheme_Anorm. There are several other internal parameters that have not been discussed in this document. In the future, it is anticipated that some of these will be made available to users.

\section{Advanced Usage of ML and Epetra}

Class ML_Epetra::MultiLevelOperator is defined in a header file, that must be included as \#include "ml_epetra_operator.h"

Users may also need to includeml_config.h, Epetra_Operator.h, Epetra_MultiVector.h, Epetra_LinearProblem.h, Aztec00.h. Check the EpeTrA and AztecOO documentation for more details.

Let $\mathrm{A}$ be an Epetra_RowMatrix for which we aim to construct a preconditioner, and let ml_handle be the structure ML requires to store internal data (see Section 7), created with the instruction

\section{ML_Create (\&ml_handle,N_levels);}

where N_levels is the specified (maximum) number of levels. As already pointed out, ML can accept in input very general matrices. Basically, the user has to specify the number of local rows, and provide a function to update the ghost nodes (that is, nodes requires in the matrix-vector product, but assigned to another process). For Epetra matrices, this is done by the following function

EpetraMatrix2MLMatrix(ml_handle, 0, \&A); 
and it is important to note that $\mathbf{A}$ is not converted to ML format. Instead, EpetraMatrix2MLMatrix defines a suitable getrow function (and other minor data structures) that allows ML to work with A.

Let agg_object a ML_Aggregate pointer, created using

ML_Aggregate_Create(\&agg_object);

At this point, users have to create the multilevel hierarchy, define the aggregation schemes, the smoothers, the coarse solver, and create the solver. Then, we can finally create the ML_Epetra::MultiLevelOperator object

ML_Epetra: :MultiLevelOperator MLop(ml_handle,comm, map, map);

(map being the Epetra_Map used to create the matrix) and set the preconditioning operator of our AzTECOO solver,

Epetra_LinearProblem Problem(A,\&x,\&b);

Aztec00 Solver (Problem);

solver.SetPrecOperator (\&MLop);

where $\mathrm{x}$ and $\mathrm{b}$ are Epetra_MultiVector's defining solution and right-hand side. The linear problem can now be solved as, for instance,

Solver.SetAztecOption( AZ_solver, AZ_gmres );

solver.Iterate (Niters, 1e-12);

\section{Using ML without Epetra}

\subsection{Creating a ML matrix: Single Processor}

Matrices are created by defining some size information, a matrix-vector product and a getrow function (which is used to extract matrix information). We note that EPETRA and AzTEC users do not need to read this (or the next) section as there are special functions to convert EPETRA objects and AzTEC matrices to ML matrices (see Section 4.2). Further, functions for some common matrix storage formats (CSR \& MSR) already exist within ML and do not need to be rewritten ${ }^{11}$.

Size information is indicated via ML_Init_Amatrix. The third parameter in the Figure 2 invocation indicates that a matrix with nlocal rows is being defined. The fourth parameter gives the vector length of vectors that can be multiplied with this matrix. Additionally, a data pointer, A_data, is associated with the matrix. This pointer is passed back into the matrix-vector product and getrow functions that are supplied by the user. Finally, the number ' 0 ' indicates at what level within the multigrid hierarchy the matrix is to be stored. For discussions within this document, this is always ' 0 '. It should be noted that there appears to be some redundant information. In particular, the number of rows and the vector length in ML_Init_Amatrix should be the same number as the discretization matrices are square. Cases where these 'apparently' redundant parameters might be set differently are not discussed in this document.

\footnotetext{
${ }^{11}$ The functions CSR_matvec, CSR_getrows, MSR_matvec and MSR_getrows can be used.
} 
The function ML_Set_Amatrix_Matvec associates a matrix-vector product with the discretization matrix. The invocation in Figure 2 indicates that the matrix-vector product function usermatvec is associated with the matrix located at level ' 0 ' of the multigrid hierarchy. The signature of user_matvec is

int user_matvec(void *A_data, int in_length, double $\mathrm{p}[]$, int out_length, double ap [])

where A_data is the user-defined data pointer specified in the ML_Init_Amatrix, $\mathrm{p}$ is the vector to apply to the matrix, in_length is the length of this vector, and ap is the result after multiplying the discretization matrix by the vector $p$ and out_length is the length of ap.

Finally, ML_Set_Amatrix_Getrow associates a getrow function with the discretization matrix. This getrow function returns nonzero information corresponding to specific rows. The invocation in Figure 2 indicates that a user supplied function user_getrow is associated with the matrix located at level ' 0 ' of the multigrid hierarchy and that this matrix contains nlocal_allcolumns columns and that no communication (NULL) is used (discussed in the next section). It again appears that some redundant information is being asked as the number of columns was already given. However, when running in parallel this number will include ghost node information and is usually different from the number of rows. The signature of user_getrow is

int user_getrow (void $* A_{\text {_ddata, }}$ int $\mathrm{N}_{-}$requested_rows, int requested_rows [], int allocated_space, int columns [], double values [], int row_lengths [])

where A_data is the user-defined data pointer in ML_Init_Amatrix, N_requested_rows is the number of matrix rows for which information is returned, requested_rows are the specific rows for which information will be returned, allocated_space indicates how much space has been allocated in columns and values for nonzero information. On return, the user's function should take each row in order within requested_rows and place the column numbers and the values corresponding to nonzeros in the arrays columns and values. The length of the ith requested row should appear in row_lengths [i]. If there is not enough allocated space in columns or values, this routine simply returns a ' 0 ', otherwise it returns a ' 1 '.

To clarify, these functions, one concrete example is given corresponding to the matrix:

$$
\left(\begin{array}{ccccc}
2 & -1 & & & \\
-1 & 2 & -1 & & \\
& -1 & 2 & -1 & \\
& & -1 & 2 & -1 \\
& & & -1 & 2
\end{array}\right)
$$

To implement this matrix, the following functions are defined:

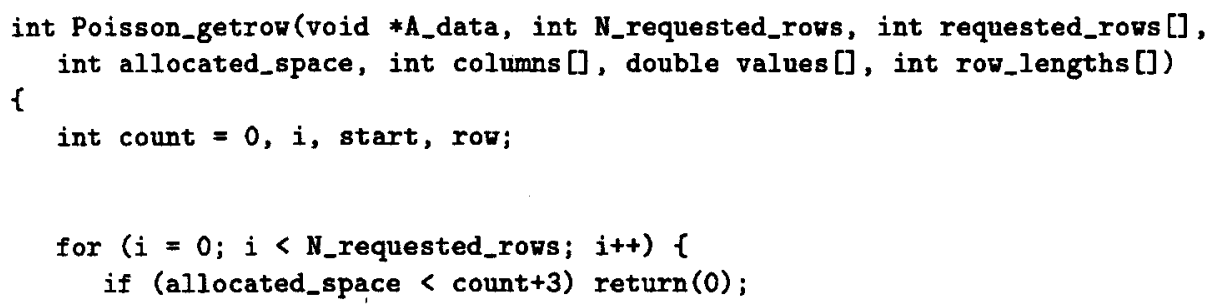




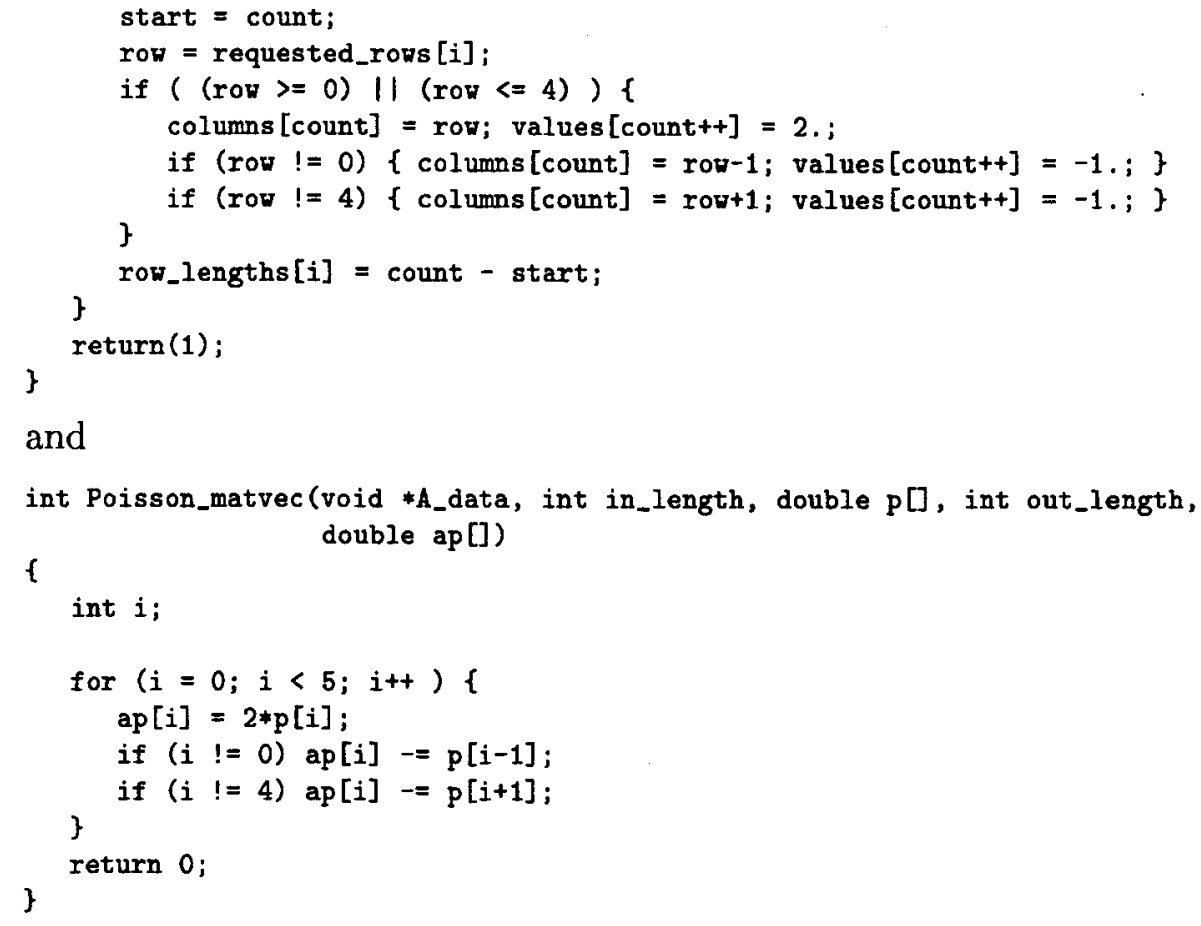

Finally, these matrix functions along with size information are associated with the fine grid discretization matrix via

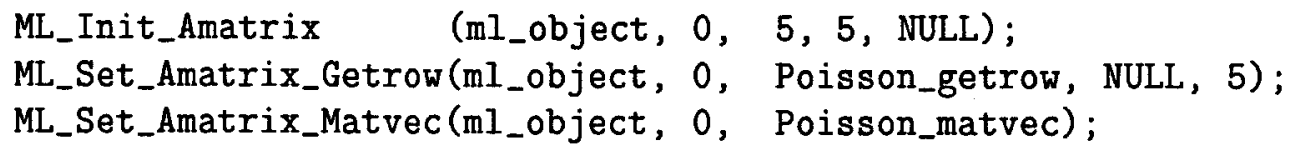

Notice that in these simple examples A_data was not used. In the next section we give a parallel example which makes use of A_data. The complete sample program can be found in the file mlguide.c within the ML code distribution.

\subsection{Creating a ML matrix: Multiple Processors}

Creating matrices in parallel requires a bit more work. In this section local versus global indexing as well as communication are discussed. In the description, we reconsider the previous example (2) partitioned over two processors. The matrix row indices (ranging from 0 to 4) are referred to as global indices and are independent of the number of processors being used. On distributed memory machines, the matrix is subdivided into pieces that are assigned to individual processors. ML requires matrices be partitioned by rows (i.e. each row is assigned to a processor which holds the entire data for that row). These matrix pieces are stored on each processor as smaller local matrices. Thus, global indices in the original matrix get mapped to local indices on each processor. In our example, we will assign global rows 0 and 4 to processor 0 and store them locally as rows 1 and 0 respectively. Global columns $0,1,3$, and 4 are stored locally as columns 1, 3, 2, and 0 . This induces the local matrix

$$
\left(\begin{array}{llll}
2 & & -1 & \\
& 2 & & -1
\end{array}\right) \text {. }
$$


Likewise, processor 1 is assigned global rows 1,2 , and 3 which are stored locally as rows 0 , 1 , and 2 respectively. Global columns $0-4$ are stored locally as columns $3,0,1,2$, and 4 inducing the local matrix

$$
\left(\begin{array}{ccccc}
2 & -1 & & & -1 \\
-1 & 2 & -1 & & \\
& -1 & 2 & -1 &
\end{array}\right) .
$$

At the present time, there are some restrictions as to what type of mappings can be used. In particular, all global rows stored on a processor must be mapped from 0 to $k-1$ where $k$ is the number of rows assigned to this processor. This row mapping induces a partial column mapping. Any additional columns must be mapped with consecutive increasing numbers starting from $k$.

ML has no notion of global indices and uses only the local indices. In most cases, another package or application already mapped the global indices to local indices and so ML works with the existing local indices. Specifically, the parallel version of user_getrow and user_matvec should correspond to each processor's local matrix. This means that when giving the column information with ML_Set_Amatrix_Getrow, the total number of columns in the local matrix should be given and that when row $k$ is requested, user_getrow should return the $k^{\text {th }}$ local row using local column indices. Likewise, the matrix-vector product takes a local input vector and multiplies it by the local matrix. It is important to note that this local input vector does not contain ghost node data (i.e. the input vector is of length nlocal where nlocal is the number of matrix rows). Thus, user matvec must perform the necessary communication to update ghost variables. When invoking ML_Init_Amatrix, the local number of rows should be given for the number of rows and the vector length ${ }^{12}$. A specific communication function must also be passed into ML when supplying the getrow function so that ML can determine how local matrices on different processors are 'glued' together. The signature of the communication function is

int user_comm(double $x[]$, void *Adata)

where $A_{-}$data is the user-defined data pointer specified in the ML_Init_Amatrix and $\mathrm{x}$ is a vector of length nlocal_allcolumns specified in ML_Set_Amatrix_Getrow. This parameter should be set to the total number of matrix columns stored on this processor. On input, only the first nlocal elements of $x$ are filled with data where nlocal is the number of rows/columns specified in ML_Init_Amatrix. On output, the ghost elements are updated to their current values (defined on other processors). Thus, after this function a local matrixvector product could be properly performed using $\mathbf{x}$. To make all this clear, we give the new functions corresponding to our two processor example.

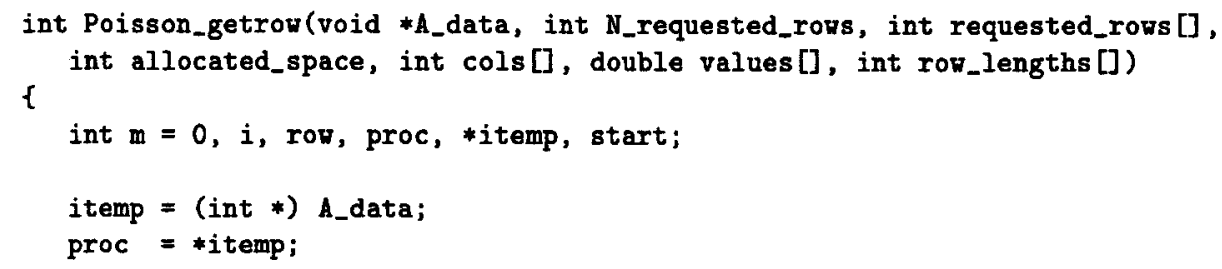

\footnotetext{
${ }^{12}$ In contrast to ML_Set_Amatrix_Getrow in which the number of local columns are given (including those that correspond to ghost variables), ML_Init_Amatrix does not include ghost variables and so both size parameters should be the number of local rows.
} 


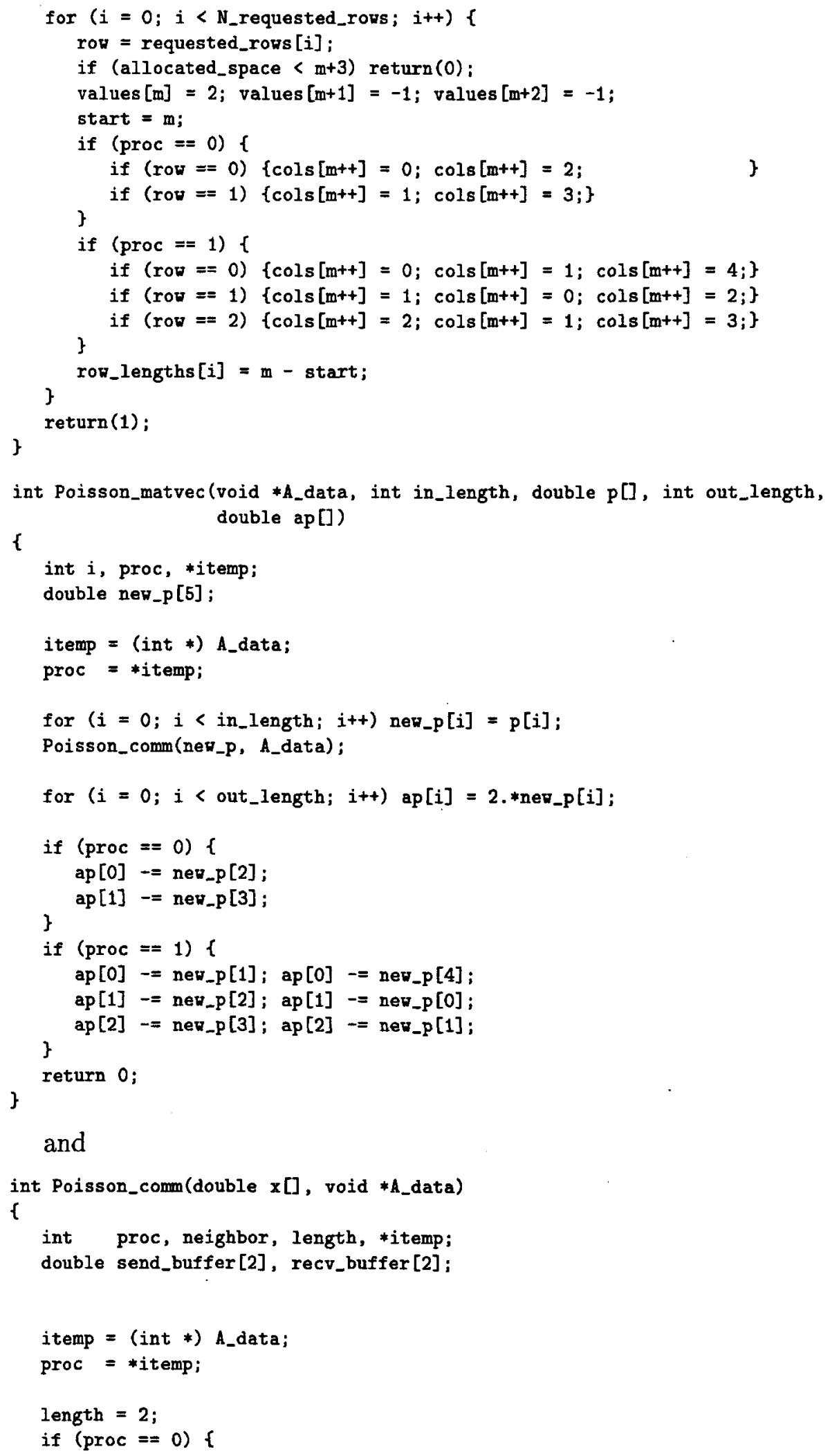




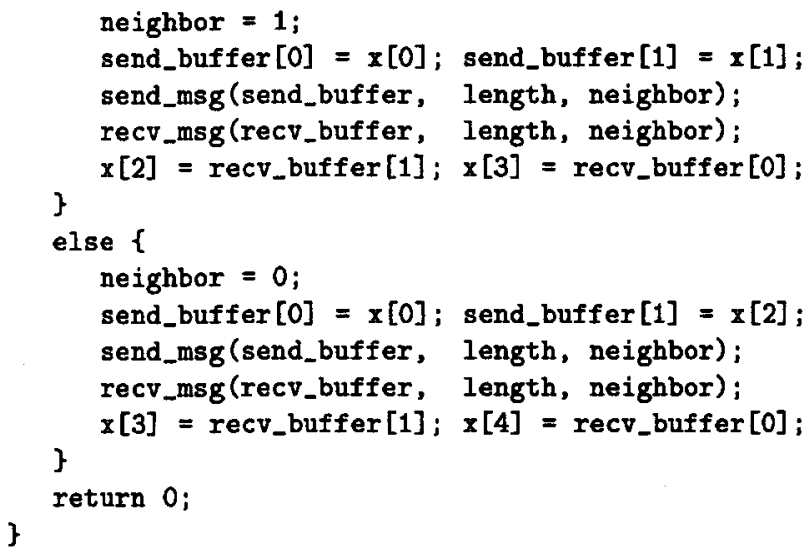

Finally, these matrix functions along with size information are associated with the fine grid discretization matrix via

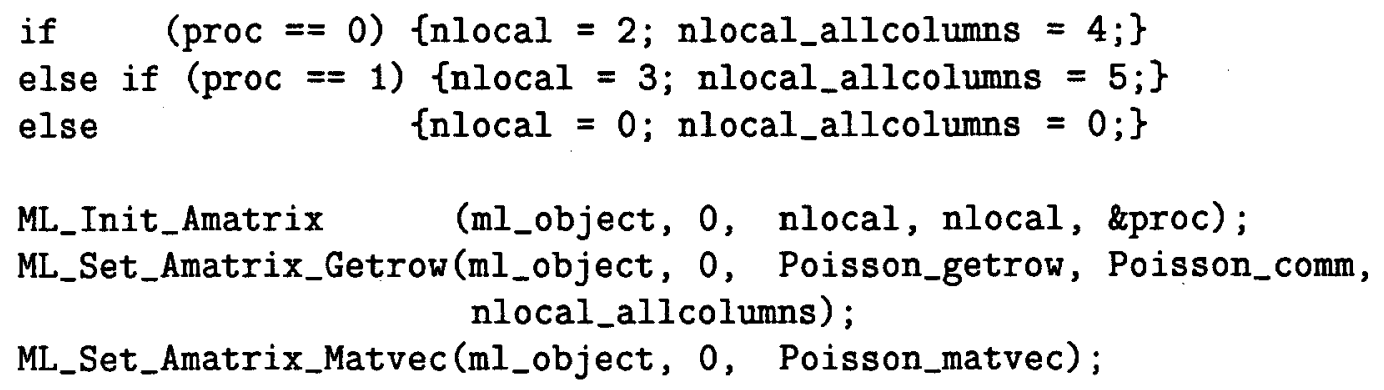

\section{Visualization Capabilities}

ML supports limited capabilities for the visualization of the aggregates, with an interface to OpenDX. Currently, only Uncoupled, METIS and ParMETIS aggregation routines can dump files in OpenDX format.

The procedure to create the OpenDX input files is as follows:

1. Add the following line after the creation of the ML_Aggregate object

$$
\text { ML_Aggregate_Viz_Stats_Setup( ag, MaxMgLevels ); }
$$

where MaxMgLevels is the maximum number of levels (this is the same value used to create the ML object).

2. Create the multilevel hierarchy;

3. Write OpenDX file using the instruction

ML_Aggregate_Visualize( $\mathrm{ml}$, ag, MaxMgLevels, $x, y, z$, option, filename);

where $\mathrm{ml}$ is the $\mathrm{ML}$ object, ag the ML_Aggregation object, and $\mathrm{x}, \mathrm{y}, \mathrm{z}$ are double vectors, whose size equals the number of local nodes in the fine grid, containing the coordinates of fine grids nodes. option is an integer value defined so that: 
- option = 1 : solution of 1D problem ( $y$ and $z$ can be NULL);

- option $=2$ : solution of $2 \mathrm{D}$ problems ( $z$ can be NULL);

- option $=3$ : solution of $3 \mathrm{D}$ problems.

Processor $X$ will write its own file, filename_levelY_procX, where $Y$ is the level. filename can be set to NULL (default value of .graph will be used in this case).

Note that, as in smoothed aggregation there is no grid for coarser levels, ML_Aggregate_Visualize needs to assign to each aggregate a set of coordinates. This is done by computing the center of gravity of each aggregates (starting from the fine grid, up to the coarsest level).

4. Deallocate memory using

ML_Aggregate_Viz_Stats_Clean( ag, MaxMgLevels )'.

At this point, one should copy file viz_aggre.net and viz_aggre.cfg (located in \$ML_HOME/util/) in the directory where the output files are located, and run OpendDX with the instruction

$\% \mathrm{dx}$-edit viz_aggre.net

Other instructions are reported in file \$ML_HOME/util/viz_aggre.README. An example of code can be found in file \$ML_HOME/examples/ml_aztec_simple_METIS.c. 


\section{ML Functions}

Prototype

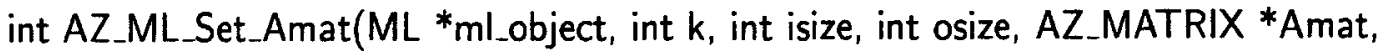
int *proc_config)

\section{Description}

Create an ML matrix view of an existing AzTECmatrix and store it within the 'ml_object' context.

\section{Parameters}

$\begin{array}{ll}\text { ml_object } & \begin{array}{l}\text { On input, ML object pointer (see ML_Create). On output, the } \\ \text { discretization matrix of level k is the same as given by Amat. }\end{array} \\ k & \text { On input, indicates level within ml_object hierarchy (should be be- } \\ \text { tween } 0 \text { and Nlevels }{ }^{\dagger}-1 \text { ). } \\ \text { On input, the number of local rows in the submatrix stored on this } \\ \text { processor. } \\ \text { On input, the number of columns in the local submatrix stored on } \\ \text { this processor not including any columns associated with ghost un- } \\ \text { knowns. } \\ \text { On input, an AzTECdata structure representing a matrix. See the } \\ \text { AzTECUser's Guide. }\end{array}$

Prototype

void AZ_set_ML_preconditioner(AZ_PRECOND **Precond, AZ_MATRIX *Amat, $M L *$ ml_object, int options[]) 
Description

Associate the multigrid $\mathrm{V}$ cycle method defined in ml_object with an AzTECpreconditioner. Thus, when Precond and options are passed into the AzTECiterative solver, it will invoke the $\mathrm{V}$ cycle multigrid algorithm described by ml_object.

\section{Parameters}

Precond

On input, an AzTECdata structure representing a preconditioner. On output, the multigrid V cycle method described by ml_object will be associated with this preconditioner. See the AztecUser's Guide.

Amat On input, an AzTECdata structure representing a matrix. See the AztecUser's Guide.

ml_object

On input, ML object pointer (see ML_Create) representing a V cycle multigrid method.

options

On input, an AzTecdata structure representing user chosen options.

On output, set appropriately for multigrid $\mathrm{V}$ cycle preconditioner.

Prototype

int ML_Aggregate_Create(ML_Aggregate **agg_object)

\section{Description}

Create an aggregate context (or handle). This instance will be used in all subsequent function invocations that set aggregation options.

\section{Parameters}

agg_object

On input, a pointer to a noninitialized ML_Aggregate object pointer.

On output, points to an initialized ML_Aggregate object pointer. 
Prototype

int ML_Aggregate_Destroy(ML_Aggregate **agg_object)

\section{Description}

Destroy the aggregate context, agg_object, and delete all memory allocated by ML in building and setting the aggregation options.

\section{Parameters}

agg_object

On input, aggregate object pointer (see ML_Aggregate_Create). On output, all memory allocated by ML and associated with this context is freed.

Prototype

int ML_Aggregate_Set_CoarsenScheme_Coupled(ML_Aggregate *agg_object)

\section{Description}

Set the aggregate coarsening scheme to be used as 'coupled' (see Section 9).

\section{Parameters}

agg_object

On input, aggregate object pointer (see ML_Aggregate_Create). On output, the 'coupled' aggregation will be used for automatic coarsening.

\section{Prototype}

int ML_Aggregate_Set_CoarsenScheme_MIS(ML_Aggregate *agg_object) 


\section{Description}

Set the aggregate coarsening scheme to be used as 'MIS' (see Section 9).

Parameters

agg_object

On input, aggregate object pointer (see ML_Aggregate_Create). On output, the 'MIS' aggregation will be used for automatic coarsening.

Prototype

int ML_Aggregate_Set_CoarsenScheme_Uncoupled(ML_Aggregate *agg_object)

\section{Description}

Set the aggregate coarsening scheme to be used as 'uncoupled' (see Section 9).

\section{Parameters}

agg_object

On input, aggregate object pointer (see ML_Aggregate_Create). On output, the 'uncoupled' aggregation will be used for automatic coarsening.

\section{Prototype}

int ML_Aggregate_Set_CoarsenScheme_METIS(ML_Aggregate *agg_object)

\section{Description}

Set the aggregate coarsening scheme to be used as 'METIS (see Section 9). 


\section{Parameters}

agg_object

On input, aggregate object pointer (see ML_Aggregate_Create). On output, the 'METIS' aggregation will be used for automatic coarsening.

Prototype

int ML_Aggregate_Set_CoarsenScheme_ParMETIS(ML_Aggregate *agg_object)

\section{Description}

Set the aggregate coarsening scheme to be used as 'ParMETIS (see Section 9).

\section{Parameters}

agg_object

On input, aggregate object pointer (see ML_Aggregate_Create). On output, the 'ParMETIS' aggregation will be used for automatic coarsening.

\section{Prototype}

int ML_Aggregate_Set_DampingFactor( ML_Aggregate *ag, double factor)

\section{Description}

Set the damping factor used within smoothed aggregation. In particular, the interpolation operator will be generated by

$$
P=\left(I-\frac{\omega}{\tilde{\rho}} A\right) P_{t}
$$

where $A$ is the discretation matrix, $\omega$ is the damping factor (default is $\frac{4}{3}$ ), $\rho$ is an estimate of the spectral radius of $A$, and $P_{t}$ are the seed vectors (tentative prolongator). 


\section{Parameters}

agg_object

On input, aggregate object pointer (see ML_Aggregate_Create). On output, the damping factor is set to factor.

factor

On input, damping factor that will be associated with this aggregation object.

Prototype

int ML_Aggregate_Set_MaxCoarseSize( ML_Aggregate *agg_object, int size )

Description

Set the maximum coarsest mesh to 'size'. No further coarsening is performed if the total number of matrix equations is less than this 'size' (see Section 8).

\section{Parameters}

agg_object

On input, aggregate object pointer (see ML_Aggregate_Create). On output, the coarsest mesh size will be set.

size

On input, size indicating the maximum coarsest mesh size.

\section{Prototype}

int ML_Aggregate_Set_NullSpace(ML_Aggregate *agg_object, int num_PDE_eqns, int null_dim,

double *null_vect, int leng)

\section{Description}

Set the seed vectors (rigid body mode vectors) to be used in smoothed aggregation. Also indicate the number of degrees of freedom (DOF) per node so that the aggregation algorithm can group them together. 


\section{Parameters}

agg_object

On input, an ML_Aggregate object pointer created by invoking ML_Aggregate_Create. On output, the seed vectors and DOFs per node are set to null_vect and num_PDE_eqns respectively.

num_PDE_eqns

On input, indicates number of equations that should be grouped in blocks when performing the aggregation. This guarantees that different DOFs at a grid point remain within the same aggregate.

null_dim

On input, number of seed vectors that will be used when creating the smoothed aggregation grid transfer operator.

null_vect

On input, the seed vectors are given in sequence. Each processor gives only the local components residing on the processor. If null, default seed vectors are used.

leng On input, the length of each seed vector.

Prototype

int ML_Aggregate_Set_SpectralNormScheme_Calc( ML_Aggregate *ag )

\section{Description}

Set the method to be used for estimating the spectral radius of $A$ (the discretization matrix) to be conjugate gradient. This spectral radius estimate is used when smoothing the initial prolongation operator (see ML_Aggregate_Set_DampingFactor).

\section{Parameters}

agg_object

On input, aggregate object pointer (see ML_Aggregate_Create). On output, the spectral radius estimate will be determined by a conjugate gradient routine.

\section{Prototype}

int ML_Aggregate_Set_SpectralNormScheme_Anorm( ML_Aggregate *ag) 


\section{Description}

Set the method to be used for estimating the spectral radius of $A$ (the discretization matrix) to be the infinity norm. This spectral radius estimate is used when smoothing the initial prolongation operator (see ML_Aggregate_Set_DampingFactor).

Parameters

agg_abject

On input, aggregate object pointer (see ML_Aggregate_Create). On output, the spectral radius estimate will be taken as the infinity norm of the matrix.

Prototype

int ML_Aggregate_Set_Threshold(ML_Aggregate *agg_object, double tolerance)

\section{Description}

Set the drop tolerance used when creating the matrix graph for aggregation. Entries in the matrix $A$ are dropped when $|A(i, j)| \leq t_{\text {tol } d} d * \sqrt{|A(i, i) A(j, j)|}$. See Section 9 for more details.

\section{Parameters}

agg_object

On input, an ML_Aggregate object pointer created by invoking ML_Aggregate_Create. On output, drop tolerance for creating the matrix graph is set.

tolerance On input, value to be used for dropping matrix entries.

\section{Prototype}

int ML_Create(ML **ml_object, int Nlevels) 


\section{Description}

Create an ML solver context (or handle). This ML instance will be used in all subsequent ML function invocations. The ML object has a notation of levels where different multigrid operators corresponding to different grid levels are stored.

\section{Parameters}

ml_object

On input, a pointer to a noninitialized ML object pointer. On output, points to an initialized ML object pointer.

Nlevels

Maximum number of multigrid levels within this ML object.

Prototype

int ML_Destroy(ML **ml_object)

\section{Description}

Destroy the ML solver context, ml_object, and delete all memory allocated by ML in building and setting options.

Parameters

ml_object

On input, ML object pointer (see ML_Create). On output, all memory allocated by ML and associated with this context is freed.

Prototype

int ML_Gen_Blocks_Aggregates(ML_Aggregate *agg_object, int $k$, int *nblocks, int **block_list) 


\section{Description}

Use aggregates to partition submatrix residing on local processor into blocks. These blocks can then be used within smoothers (see for example ML_Gen_Smoother_VBlockJacobi or ML_Gen_Smoother_VBlockSymGaussSeidel).

\section{Parameters}

$m l_{-}$object

$k$

nblocks

block_list
On input, ML object pointer (see ML_Create).

On input, indicates level within ml_object hierarchy where the aggregate information is found that defines partitioning.

On output, indicates the number of partitions.

On output, equation i resides in the block_list[i]th partition.

Prototype

int ML_Gen_Blocks_Metis(ML *ml_object, int $k$, int *nblocks, int **block_list)

\section{Description}

Use Metis to partition submatrix residing on local processor into blocks. These blocks can then be used within smoothers (see for example ML_Gen_Smoother_VBlockJacobi or ML_Gen_Smoother_VBlockSymGaussSeidel).

\section{Parameters}
ml_object
On input, ML object pointer (see ML_Create).
$k$
On input, indicates level within ml_object hierarchy where the dis- cretization matrix is found that will be partitioned.
nblocks
On input, indicates number of partitions desired on each processor.
On output, indicates the number of partitions obtained.

block_list

On output, equation i resides in the block_list[i]th partition. 


\section{Prototype}

int ML_Gen_CoarseSolverSuperLU(ML *ml_object, int k)

\section{Description}

Use SuperLU for the multigrid coarse grid solver on level $\mathrm{k}$ within ml_object and perform any initialization that is necessary.

\section{Parameters}

ml_object

On input, ML object pointer (see ML_Create). On output, the coarse grid solver of level $k$ is set to use SuperLU.

$k$

On input, indicates level within ml_object hierarchy (must be the coarsest level in the multigrid hierarchy).

\section{Prototype}

int ML_Gen_MGHierarchy_UsingAggregation(ML *ml_object, int start, int inc_or_dec,

ML_Aggregate *agg_object)

\section{Description}

Generate a multigrid hierarchy via the method of smoothed aggregation. This hierarchy includes a series of grid transfer operators as well as coarse grid approximations to the fine grid discretization operator. On completion, return the total number of multigrid levels in the newly created hiearchy.

\section{Parameters}

ml_object

On input, ML object pointer (see ML_Create). On output, coarse levels are filled with grid transfer operators and coarse grid discretizations corresponding to a multigrid hierarchy. 
start

inc_or_dec

agg_object
On input, indicates multigrid level within ml_object where the fine grid discretization is stored.

On input, ML_INCREMENT or ML_DECREMENT. Normally, set to MLINCREMENT meaning that the newly created multigrid operators should be stored in the multigrid levels: start, start+1, start+2, start+3, etc. If Set to ML_DECREMENT, multigrid operators are stored in start, start-1, start-2, etc.

On input, an initialized aggregation object defining options to the generation of grid transfer operators. If set to NULL, default values are used for all aggregation options. See ML_Aggregate_Create.

Prototype

int ML_Gen_SmootherAmesos(ML *ml_object, int $k$, int AmesosSolver, int MaxProcs)

\section{Description}

Use Amesos interface to direct solvers for the multigrid coarse grid solver on level $\mathrm{k}$ within ml_object and perform any initialization that is necessary.

\section{Parameters}

ml_object

$k$

AmesosSolver

MaxProcs
On input, ML object pointer (see ML_Create). On output, the coarse grid solver of level $\mathrm{k}$ is set to use Amesos.

On input, indicates level within ml_object hierarchy (must be the coarsest level in the multigrid hierarchy).

On input, indicates the direct solver library to use in the coarse solution. It can be: ML_AMESOS_UMFPACK, ML_AMESOS_KLU, ML_AMESOS_SUPERLUDIST, ML_AMESOS_SCALAPACK. ML_AMESOS_MUMPS,

On input, indicates maximum number of processors to use in the coarse solution (only for ML_AMESOS_SUPERLUDIST). 
Prototype

void ML_Gen_SmootherAztec(ML *ml_object, int k, int options[], double params[], int proc_config[], double status[], int N_iterations, int pre_or_post, void (*prec_fun)(double *, int *, int *, double *, AZ_MATRIX *, AZ_PRECOND *))

\section{Description}

Set the smoother (either pre or post as indicated by pre_or_post) at level $\mathrm{k}$ within the multigrid solver context to invoke AzTEC. The specific AzTECscheme is given by the AzTECarrays: options, params, proc_config, and status and AzTECpreconditioning function: precfunction.

\section{Parameters}

ml_object

$k$

options, params proc_config, status

$N_{-}$iterations

pre_or_post

prec_fun
On input, ML object pointer (see ML_Create). On output, a smoother function is associated within ml_object at level $\mathrm{k}$.

On input, indicates where the smoother function pointer will be stored within the multigrid hierarchy.

On input, AzTECarrays that determine the AzTECscheme and are used for AzTECto return information. See the AzTECUser's Guide.

On input, maximum Azteciterations within a single smoother invocation. When set to AZ_ONLY_PRECONDITIONER, only one iteration of the preconditioner is used without an outer Krylov method.

On input, ML_PRESMOOTHER or ML_POSTSMOOTHER indicating whether the smoother should be performed before or after the coarse grid correction.

On input, AzTECpreconditioning function indicating what preconditioner will be used within AzTEC. Normally, this is set to AZ_precondition. See the AzTECUser's Guide. 
int ML_Gen_Smoother_BlockGaussSeidel(ML *ml_object, int k, int pre_or_post, int ntimes, double omega, int blocksize)

\section{Description}

Set the multigrid smoother for level $\mathrm{k}$ of ml_object and perform any initialization that is necessary. When using block Gauss Seidel, the total number of equations must be a multiple of blocksize. Each consecutive group of blocksize unknowns is grouped into a block and a block Gauss Seidel algorithm is applied.

\section{Parameters}

ml_object On input, ML object pointer (see ML_Create). On output, the pre or post smoother of level $\mathrm{k}$ is set to block Gauss Seidel.

$k$

On input, indicates level within ml_object hierarchy (should be between 0 and Nlevels ${ }^{\dagger}-1$ ). ML_ALL_LEVELS sets the smoothing on all levels in ml_object.

pre_or_post On input, ML_PRESMOOTHER or ML_POSTSMOOTHER indicating whether the pre or post smoother is to be set.

ntimes $\quad$ On input, sets the number of block Gauss Seidel iterations that will be performed.

omega On input, sets the damping parameter to be used during this block Gauss Seidel smoothing.

blocksize On input, sets the size of the blocks to be used during block Gauss Seidel smoothing.

Prototype

int ML_Gen_Smoother_GaussSeidel(ML *ml_object, int k, int pre_or_post, int ntimes, double omega) 


\section{Description}

Set the multigrid smoother for level $\mathrm{k}$ of ml_object and perform any initialization that is necessary.

\section{Parameters}

ml_object

$k$

pre_or_post

ntimes

omega
On input, ML object pointer (see ML_Create). On output, the pre or post smoother of level $\mathrm{k}$ is set to Gauss Seidel.

On input, indicates level within ml_object hierarchy (should be between 0 and Nlevels ${ }^{\dagger}-1$ ). ML_ALL_LEVELS sets the smoothing on all levels in ml_object.

On input, ML_PRESMOOTHER or ML_POSTSMOOTHER indicating whether the pre or post smoother is to be set.

On input, sets the number of Gauss Seidel iterations that will be performed.

On input, sets the damping parameter to be used during this Gauss Seidel smoothing.

Prototype

int ML_Gen_Smoother_Jacobi(ML *ml_object, int $k$, int pre_or_post, int ntimes, double omega)

\section{Description}

Set the multigrid smoother for level $\mathrm{k}$ of ml_object and perform any initialization that is necessary.

\section{Parameters}

ml_object

$k$
On input, ML object pointer (see ML_Create). On output, the pre or post smoother of level $\mathrm{k}$ is set to Jacobi.

On input, indicates level within ml_object hierarchy (should be between 0 and Nlevels ${ }^{\dagger}-1$ ). ML_ALL_LEVELS sets the smoothing on all levels in ml_object. 

cating whether the pre or post smoother is to be set.

ntimes

On input, sets the number of Jacobi iterations that will be performed.

omega

On input, sets the damping parameter to be used during this Jacobi smoothing. ML_DEFAULT sets it to .5

Prototype

int ML_Gen_Smoother_SymGaussSeidel(ML *ml_object, int $k$, int pre_or_post, int ntimes, double omega)

Description

Set the multigrid smoother for level $\mathrm{k}$ of $\mathrm{ml}$ _object and perform any initialization that is necessary.

\section{Parameters}

ml_object

On input, ML object pointer (see ML_Create). On output, the pre or post smoother of level $\mathrm{k}$ is set to symmetric Gauss Seidel.

$k$

On input, indicates level within ml_object hierarchy (should be between 0 and Nlevels ${ }^{\dagger}-1$ ). ML_ALL_LEVELS sets the smoothing on all levels in ml_object.

pre_or_post

On input, ML_PRESMOOTHER or ML_POSTSMOOTHER indicating whether the pre or post smoother is to be set.

ntimes

On input, sets the number of symmetric Gauss Seidel iterations that will be performed.

omega On input, sets the damping parameter to be used during this symmetric Gauss Seidel smoothing. 
int ML_Gen_Smoother_VBlockJacobi(ML *ml_object, int $k$, int pre_or_post, int ntimes, double omega, int nBlocks, int *blocklndices)

\section{Description}

Set the multigrid smoother for level $\mathbf{k}$ of ml_object and perform any initialization that is necessary. A block Jacobi smoothing algorithm will be used where the size of the blocks can vary and is given by nBlocks and blockIndices (see ML_Gen_Blocks_Aggregates and ML_Gen_Blocks_Metis).

\section{Parameters}

ml_object

On input, ML object pointer (see ML_Create). On output, the pre or post smoother of level $\mathrm{k}$ is set to variable block Jacobi.

$k$

On input, indicates level within ml_object hierarchy (should be between 0 and Nlevels ${ }^{\dagger}-1$ ). ML_ALL_LEVELS sets the smoothing on all levels in ml_object.

pre_or_post

On input, ML_PRESMOOTHER or ML_POSTSMOOTHER indicating whether the pre or post smoother is to be set.

ntimes

On input, sets the number of block Jacobi iterations that will be performed.

omega

On input, sets the damping parameter to be used during this block Jacobi smoothing.

nBlocks

On input, indicates the total number of block equations in matrix.

blockIndices

On input, blockIndices[i] indicates block to which ith element belongs.

\section{Prototype}

int ML_Gen_Smoother_VBlockSymGaussSeidel(ML *ml_object, int k, int pre_or_post, int ntimes, double omega, int nBlocks, int *blocklndices) 


\section{Description}

Set the multigrid smoother for level $\mathrm{k}$ of ml_object and perform any initialization that is necessary. A block Gauss Seidel smoothing algorithm will be used where the size of the blocks can vary and is given by nBlocks and blockIndices (see ML_Gen_Blocks_Aggregates and ML_Gen_Blocks_Metis).

\section{Parameters}

ml_object

$k$

pre_or_post

ntimes

omega

nBlocks

blockIndices
On input, ML object pointer (see ML_Create). On output, the pre or post smoother of level $k$ is set to variable block symmetric Gauss Seidel.

On input, indicates level within ml_object hierarchy (should be between 0 and Nlevels ${ }^{\dagger}-1$ ). ML_ALL_LEVELS sets the smoothing on all levels in ml_object.

On input, ML_PRESMOOTHER or ML_POSTSMOOTHER indicating whether the pre or post smoother is to be set.

On input, sets the number of block symmetric Gauss Seidel iterations that will be performed.

On input, sets the damping parameter to be used during this block symmetric Gauss Seidel smoothing.

On input, indicates the total number of block equations in matrix.

On input, blockIndices[i] indicates block to which ith element belongs.

\section{Prototype}

int ML_Gen_Solver(ML *ml_object, int scheme, int finest_level, int coarsest_level)

\section{Description}

Initialize the ML solver context, ml_object, so that it is ready to be used in a solve. ML_Gen_Solver should be called after the multigrid cycle is fully specified but before ML_Iterate or ML_Solve_MGV is invoked. 
Parameters

ml_object

scheme

finest_level

coarsest_level
On input, ML object pointer (see ML_Create). On output, all necessary initialization is completed.

On input, must be set to ML_MGV indicating a multigrid $\mathrm{V}$ cycle is used.

On input, indicates the location within ml_object where the finest level is stored. Normally, this is ' 0 '.

On input, indicates location within ml_object where the coarsest grid is stored. When doing smoothed aggregation, this can be determined using the total number of multigrid levels returned by ML_Gen_MGHierarchy_UsingAggregation.

Prototype

int ML_Get_Amatrix(ML *ml_object, int k, ML_Operator **matrix)

\section{Description}

Set *matrix to point to the discretization matrix associated at level $\mathrm{k}$ within the multigrid solver context ml_object. This pointer can then be passed into functions like:

ML_Operator_Apply, ML_Operator_Get_Diag, and ML_Operator_Getrow.

\section{Parameters}

ml_object

$k$

matrix
On input, ML object pointer (see ML_Create).

On input, indicates which level within the multigrid hierarchy should be accessed.

On output, ${ }^{*}$ matrix points to the discretization matrix at level $\mathrm{k}$ within the multigrid hierarchy. This pointer can then be passed into the functions ML_Operator_Apply, ML_Operator_Get_Diag, and ML_Operator_Getrow. 
int ML_Init_Amatrix $\left(\mathrm{ML}{ }^{*}\right.$ ml_object, int $k$, int ilen, int olen, void *data)

\section{Description}

Set the size information for the discretization matrix associated at level $k$ within ml_object. Additionally, associate a data pointer that can be used when writting matrix-vector product and matrix getrow functions.

\section{Parameters}
ml_object
On input, ML object pointer (see ML_Create). On output, size information is associated with the discretization matrix at level $\mathrm{k}$.
$k$
On input, indicates where discretization size information will be stored within the multigrid hierarchy.
ilen On input, the number of local rows in the submatrix stored on this processor.
olen On input, the number of columns in the local submatrix stored on this processor not including any columns associated with ghost un- knowns.
data On input, a data pointer that will be associated with the discretiza- tion matrix and could be used for matrix-vector product and matrix getrow functions.

Prototype

int ML_Iterate $(\mathrm{ML}$ *ml_object, double *sol, double *rhs)

\section{Description}

Iterate until convergence to solve the linear system using the multigrid V cycle defined within ml_object. 


\section{Parameters}

ml_object

sol

rhs
On input, ML object pointer (see ML_Create).

On input, a vector containing the initial guess for the linear system contained in ml_object. On output, the solution obtained by performing repeated multigrid $\mathrm{V}$ cycles.

On input, a vector contain the right hand side for the linear system contained in ml_object.

Prototype

int ML_Operator_Apply(ML_Operator *A, int in_length, double $p[]$, int out_length, double ap[])

\section{Description}

Invoke a matrix-vector product using the ML_Operator A. That is perform $a p=A * p$. Any communication or ghost variables work needed for this operation is also performed.

\section{Parameters}

$A$

in_length

$p$

out_length

$a p$
On input, an ML_Operator (see ML_Get_Amatrix).

On input, length of vector $p$ (not including ghost variable space).

On input, vector which will be multiplied by $A$.

On input, length of vector $a p$.

On output, vector containing result of $A * p$.

\section{Prototype}

int ML_Operator_Get_Diag(ML_Operator ${ }^{*} A$, int length, double **diag) 


\section{Description}

Get the diagonal of the ML_Operator A (which is assumed to be square).

Parameters

A On input, an ML_Operator (see ML_Get_Amatrix).

length On input, number of diagonal elements wanted.

diag On output, sets a pointer to an array containing the diagonal elements. NOTE: this is not a copy but in fact a pointer into an ML data structure. Thus, this array should not be freed.

Prototype

int ML_Operator_Getrow(ML_Operator *A, int N_requested_rows, int requested_rows], int allocated_space, int columns[], double values[], int row_lengths[])

\section{Description}

Get a row (or several rows) from the ML_Operator A. If there is not enough space in columns and values to hold the nonzero information, this routine returns a ' 0 '. Otherwise, a ' 1 ' is returned.

\section{Parameters}

$A$

N_requested_rows

requested_rows

allocated_space

columns
On input, an ML_Operator (see ML_Get_Amatrix).

On input, number of matrix rows for which information is returned.

On input, specific rows for which information will be returned.

On input, length of columns and values.

On output, the column numbers of each nonzero within each row requested in requested_rows (where column numbers associated with requested_rows [i] appear before column numbers associated with requested_rows $[j]$ with $i<j$ ). 
values

row_lengths
On output, the nonzero values of each nonzero within each row requested in requested_rows (where nonzero values associated with requested_rows [i] appear before nonzero values associated with requested_rows $[j]$ with $i<j$ ).

On output, row_lengths [i] indicates the number of nonzeros in row i.

Prototype

int ML_Set_Amatrix_Getrow $\left(M L{ }^{*}\right.$ ml_object, int $k_{\text {, int }}\left({ }^{*}\right.$ getrow)(void *, int , int* , int, int*, double*, int*), int (*comm $)($ double *vec, void *data), int comm_vec_leng)

\section{Description}

Set the matrix getrow function for the discretization matrix associated at level $\mathrm{k}$ within the multigrid solver context ml_object.

\section{Parameters}

ml_object

$k$

getrow

comm
On input, ML object pointer (see ML_Create). On output, matrix getrow function is associated with the discretization matrix at level k.

On input, indicates where the matrix getrow function pointer will be stored within the multigrid hierarchy.

On input, a function pointer to the user-defined matrix getrow function. See Section 11.1.

On input, a function pointer to the user-defined communication function. See Section 11.2.

\section{Prototype}

int ML_Set_Amatrix_Matvec $\left(M L{ }^{*}\right.$ ml_object, int $k$, int $\left({ }^{*}\right.$ matvec $)($ void *, int, double *, int, double $\left.{ }^{*}\right)$ ) 


\section{Description}

Set the matrix-vector product function for the discretization matrix associated at level $\mathrm{k}$ within the multigrid solver context ml_object.

\section{Parameters}

\begin{tabular}{|c|c|}
\hline ml_object & $\begin{array}{l}\text { On input, ML object pointer (see ML_Create). On output, matrix- } \\
\text { vector product function is associated with the discretization matrix } \\
\text { at level } \mathrm{k} \text {. }\end{array}$ \\
\hline$k$ & $\begin{array}{l}\text { On input, indicates where the matrix-vector product function } \\
\text { pointer is stored within the multigrid hierarchy. }\end{array}$ \\
\hline at & , a function pointer to the user-defined \\
\hline
\end{tabular}
uct function. See Section 11.1

Prototype

int ML_Set_ResidualOutputFrequency(ML *ml_object, int output_freq)

\section{Description}

Set the output frequency of residual information. ML_Iterate prints the two norm of the residual every output_freq iterations.

\section{Parameters}

ml_object

On input, ML object pointer (see ML_Create). On output, residual printing frequency is set.

output_freq On input, value to use for printing frequency.

\section{Prototype}


int ML_Set_Smoother(ML *ml_object, int $k$, int pre_or_post, void *data, int $\left({ }^{*}\right.$ func $)\left(\right.$ void *, int, double *, int, double $\left.{ }^{*}\right)$, char *label)

\section{Description}

Set the smoother (either pre or post as indicated by pre_or_post) at level $\mathrm{k}$ within the multigrid solver context to invoke the user-defined function 'func' and pass in the data pointer 'data'.

\section{Parameters}

ml_object

$k$

pre_or_post

label
On input, $\mathbf{M L}$ object pointer (see ML_Create). On output, a smoother function is associated within ml_object at level $\mathbf{k}$.

On input, indicates where the smoother function pointer will be stored within the multigrid hierarchy.

On input, ML_PRESMOOTHER or ML_POSTSMOOTHER indicating whether the smoother should be performed before or after the coarse grid correction.

data On input, a data pointer that will be passed into the user-defined function 'func'.

func On input, smoothing function to be used at level $\mathrm{k}$ when performing a multigrid $\mathrm{V}$ cycle. The specific signature and details of this function are given in Section 8.

On input, a character string to be associated with Smoother. This string is printed by some routines when identifying the method.

Prototype

int ML_Set_Tolerance $\left(M L{ }^{*}\right.$ ml_object, double tolerance) 
Set the convergence criteria for MLIterate. Convergence is declared when the 2-norm of the residual is reduced by 'tolerance' over the initial residual. This means that if the initial residual is quite small (i.e. the initial guess corresponds quite closely with the true solution), ML_Iterate might continue to iterate without recognizing that the solution can not be improved due to round-off error. Note: the residual is always computed after performing presmoothing on the finest level (as opposed to at the beginning or end of the iteration). Thus, the true residual should be a little bit better than the one used by ML.

\section{Parameters}

ml_object

On input, ML object pointer (see ML_Create). On output, tolerance is set for convergence of ML_Iterate.

tolerance $\quad$ On input, value to use for convergence tolerance.

Prototype

int ML_Solve_MGV(ML *ml_object, double *din, double *dout)

\section{Description}

Perform one multigrid V cycle iteration to the solve linear system defined within ml_object.

$\begin{array}{ll}\text { Parameters } & \\ \text { ml_object } & \text { On input, ML object pointer (see ML_Create). } \\ \text { din } & \begin{array}{l}\text { On input, the right hand side vector to be used when performing } \\ \text { multigrid. }\end{array} \\ \text { dout } & \begin{array}{l}\text { On output, an approximate solution obtained after one multigrid V } \\ \text { cycle. }\end{array}\end{array}$

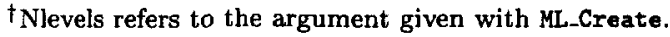




\section{References}

[1] A. Brandt, Multi-level Adaptive Solutions to Boundary-Value Problems, Math. Comp., 31 (1977), pp. 333-390.

[2] T. DAVIS, UMFPACK home page. http://www.cise.ufl.edu/research/sparse/umfpack, 2003.

[3] J. W. Demmel, J. R. Gilbert, And X. S. Li, SuperLU Users' Guide, 2003.

[4] Free Software Foundation, Autoconf Home Page.

http://www.gnu.org/software/autoconf.

[5] —_, Automake Home Page. http://www.gnu.org/software/automake.

[6] W. HackBusch, Multi-grid Methods and Applications, Springer-Verlag, Berlin, 1985.

[7] - Iterative Solution of Large Sparse Linear Systems of Equations, Springer-Verlag, Berlin, 1994.

[8] M. A. Heroux, Trilinos home page. http://software.sandia.gov/trilinos.

[9] M. A. Heroux, IFPACK Reference Manual, 2.0 ed., 2003.

http://software.sandia.gov/trilinos/packages/ifpack/doxygen/latex/IfpackReferenceManual.pdf.

[10] M. M. A. Heroux, Trilinos Tutorial, 3.1 ed., 2004.

[11] G. Karypis and V. Kumar, ParMETIS: Parallel graph partitioning and sparse matrix ordering li brary, Tech. Rep. 97-060, Department of Computer Science, University of Minnesota, 1997.

[12] _ _ METIS: Unstructured graph partitining and sparse matrix ordering sy stem, tech. rep., University of Minnesota, Department of Computer Science, 1998.

[13] T. G. Kolda and R. P. Pawlowski, Nox home page. http://software.sandia.gov/nox.

[14] I. S. D. P. R. Amestoy AND J.-Y. L'Excellent, Multifrontal parallel distributed symmetric and unsymmetric solvers, Comput. Methods in Appl. Mech. Eng., (2000), pp. 501-520.

[15] R. Tuminaro, M. Heroux, S. Hutchinson, and J. Shadid, Official Aztec user's guide: Version 2.1, Tech. Rep. Sand99-8801J, Sandia National Laboratories, Albuquerque NM, 87185, Nov 1999.

[16] R. Tuminaro And C. Tong, Parallel smoothed aggregation multigrid: Aggregation strategies on massively parallel machines, in SuperComputing 2000 Proceedings, J. Donnelley, ed., 2000.

[17] P. Vanek, M. Brezina, And J. Mandel, Convergence of Algebraic Multigrid Based on Smoothed Aggregation, Tech. Rep. report 126, UCD/CCM, Denver, CO, 1998. 
[18] P. Vanek, J. Mandel, And M. Brezina, Algebraic Multigrid Based on Smoothed Aggregation for Second and Fourth Order Problems, Computing, 56 (1996), pp. 179-196. 
Distribution list

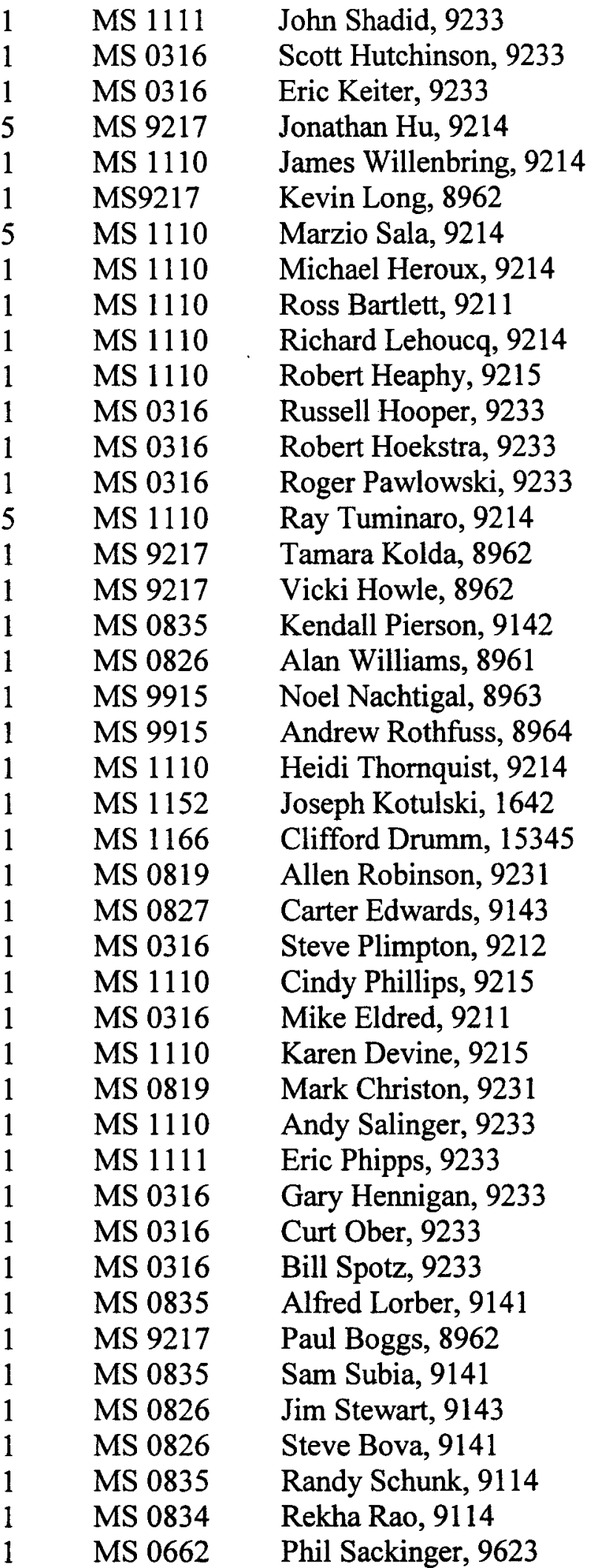




$\begin{array}{lll}1 & \text { MS 0834 } & \text { Matt Hopkins, 9114 } \\ 1 & \text { MS 0834 } & \text { Harry Moffat, 9114 } \\ 1 & \text { MS 0847 } & \text { Garth Reese, 9142 } \\ 1 & \text { MS 1110 } & \text { David Day, 9214 } \\ 1 & \text { MS 1110 } & \text { Bart van Bloemen Waanders, 9211 } \\ 1 & \text { MS 9217 } & \text { Patty Hough, 8962 } \\ & & \\ 1 & \text { MS 9018 } & \text { Central Technical Files, 8945-1 } \\ 2 & \text { MS 0899 } & \text { Technical Library, 9616 }\end{array}$

External distribution:

Ken Stanley

322 W. College St.

Oberlin $\mathrm{OH} 44074$

Matthias Heinkenschloss

Department of Computational and Applied Mathematics - MS 134

Rice University

6100 S. Main Street

Houston, TX 77005 - 1892

Dan Sorenson

Department of Computational and Applied Mathematics - MS 134

Rice University

6100 S. Main Street

Houston, TX $77005-1892$

Yousef Saad

Department of Computer Science and Engineering

University of Minnesota,

4-192 EE/CSci Building, 200 Union Street S.E.

Minneapolis, MN 55455

Kris Kampshoff

Department of Computer Science and Engineering

University of Minnesota,

EE/CSci Building, 200 Union Street S.E.

Minneapolis, MN 55455

Eric de Sturler

2312 Digital Computer Laboratory, MC-258

University of Illinois at Urbana-Champaign

1304 West Springfield Avenue

Urbana, IL 61801-2987 
Jason Cross

Box 429

St. John's University

Collegeville, MN 56321

Paul Sexton

Box 1560

St. John's University

Collegeville, MN 56321

Mike Phenow

PO Box 1392

St. John's University

Collegeville, MN 56321

Tim Davis, Assoc. Prof.

Room E338 CSE Building

P.O. Box 116120

University of Florida

Gainesville, FL 32611-6120

Padma Raghavan

Department of Computer Science and Engineering

308 Pond Laboratory

The Pennsylvania State University

University Park, PA 16802-6106

Xiaoye $\mathrm{Li}$

Lawrence Berkeley Lab

$50 \mathrm{~F}-1650$

1 Cyclotron Rd

Berkeley, CA 94720

Richard Barrett

Los Alamos National Laboratory

Mail Stop B272

Los Alamos, NM 87545

Victor Eijkhout

Department of Computer Science,

203 Claxton Complex, 1122 Volunteer Boulevard,

University of Tennessee at Knoxville,

Knoxville TN 37996, USA 
Jack Dongarra

Computer Science Department

1122 Volunteer Blvd

Knoxville, TN 37996-3450

David Keyes

Appl Phys \& Appl Math

Columbia University

200 S. W. Mudd Building

500 W. 120th Street

New York, NY, 10027

Lois Curfman McInnes

Mathematics and Computer Science Division

Argonne National Laboratory

9700 South Cass Avenue

Argonne, IL 60439

Barry Smith

Mathematics and Computer Science Division

Argonne National Laboratory

9700 South Cass Avenue

Argonne, IL 60439

Paul Hovland

Mathematics and Computer Science Division

Argonne National Laboratory

9700 South Cass Avenue

Argonne, IL 60439

Jeffrey J. Derby

CEMS Department, U. of MN

421 Washington Ave SE

Minneaplolis, MN 55455-0132

Carol Woodward

Center for Applied Scientific Computing

Lawrence Livermore National Laboratory

Box 808, L-561

Livermore, CA 94551

Craig Douglas

325 McVey Hall - CCS

Lexington, KY 40506-0045 


\section{Juan Meza}

Department Head, High Performance Computing Research

Lawrence Berkeley National Laboratory

Mail Stop 50B-2239

Berkeley, CA 9472

C.T. Kelley

Department of Mathematics, Box 8205

Center for Research in Scientific Computation

North Carolina State University

Raleigh, NC 27695-8205

Chuck Romine

Program Manager, Applied Mathematics

U.S. Department of Energy

1000 Independence Ave., SW

Washington, DC 20585-1290

Prof. Luca Formaggia

Mathematics Department

"F. Brioschi" Politecnico di Milano

Piazza L. da Vinci 32, 20133 MILANO, Italy

Prof. Alfio Quarteroni

IACS-CMCS

EPFL

CH-1015 Lausanne (VD) Switzerland

LIBRARY DOCUMENT
DO NOT DESTROY
RETURN TO
LIBRARY VAULT

